\title{
Dynamic User Grouping and Joint Resource Allocation with Multi-Cell Cooperation for Uplink Virtual MIMO Systems
}

\author{
Xiaofeng Lu, Qiang Ni, Wenna Li, Hailin Zhang
}

\begin{abstract}
This paper proposes a novel joint resource allocation algorithm combining dynamic user grouping, multi-cell cooperation and resource block (RB) allocation for single carrierfrequency division multiple access (SC-FDMA) uplink in multicell virtual MIMO systems. We first develop the dynamic multicell user grouping criteria using minimum mean square error (MMSE) equalization and adaptive modulation (AM) with bit error rate (BER) constraint. Then, we formulate and solve a new throughput maximization problem whose resource allocation includes cell selection, dynamic user grouping and RB pattern assignment. Furthermore, to reduce the computational complexity significantly, especially in the case of large numbers of users and RBs, we present an efficient iterative Hungarian algorithm based on user and resource partitions (IHA_URP) to solve the problem by decomposing the large scale problem into a series of small scale sub-problems, which can obtain close-to-optimal solution with much lower complexity. The simulation results show that our proposed joint resource allocation algorithm with dynamic multicell user grouping scheme achieves better system throughput with BER guarantee than fixed user grouping algorithm and other proposed schemes in the literature.
\end{abstract}

Index Terms-Virtual MIMO, dynamic user grouping, fairness, resource allocation, multi-cell cooperation, user and resource partitions

\section{INTRODUCTION}

$\mathbf{M}$ ULTIPLE-INPUT multiple-output (MIMO) techniques have been widely applied in increasing the spectral efficiency (SE) in various wireless communication systems [1]. However, because of the cost and size of the user equipment (UE), the application of MIMO uplink is limited by the difficulty in practical implementation at the user side, especially in a small handset. In order to deal with this problem, virtual MIMO for uplink approach [2]-[5] that assigns two or more users, each with single transmitting antenna, to the same frequency band and time slot emerged. Compared with a conventional MIMO system, virtual MIMO can obtain additional multiuser diversity gain by grouping users using well-designed strategies.

This work was supported by the National Natural Science Foundation of China (61371127 \& 61471361\& 61572389), the EU FP7 CROWN Project under Grant Number PIRSES-GA-2013-610524, the National High-Tech R\&D Program (863 Program 2015AA01A705) and the 111 Project in Xidian University of China (B08038)

Xiaofeng Lu(e-mail: luxf@xidian.edu.cn), Wenna Li and Hailin Zhang are with State Key Laboratory of Integrated Service Networks, Xidian University, China. e-mail:(luxf@xidian.edu.cn).

Qiang Ni is with School of Computing and Communications, Lancaster University, LA1 4WA, U.K. (e-mail: q.ni@lancaster.ac.uk).
Some research works have been performed on the criteria of user pairing/grouping for virtual MIMO systems. Most of these proposed criteria are derived from the channel capacity [6], [9]-[11]. In [6], $n$-user virtual MIMO channel capacity is calculated using the theory in [7], and a suboptimal pairing algorithm which selects pairing users one by one is proposed. Similarly, in [8], the decision metric which employs instantaneous receiving signal-to-noise ratio (SNR) after maximum likelihood (ML) detection is equivalent to MIMO channel capacity. In [9], J. Fan et al. analyze the receiving signal to interference plus noise ratio (SINR) after minimum mean square error (MMSE) equalization and use Shannon capacity as user scheduling criterion. Similar method is adopted for uplink virtual MIMO system with linear receiver in [10], [11]. In addition, some research works construct grouping criteria considering user fairness. Most of them apply proportional fair idea to partner user scheduling process [11], [14]-[17]. Although capacity criteria are convenient to use, they only display the performance of ideal transmission. For actual communication systems, bit error rate (BER) or symbol error rate (SER) under given system throughput is usually used as the performance metric for data transmission and user grouping at physical layer. Most of these research works are performed with linear MIMO detection such as zero forcing (ZF)/MMSE or the expansion of successive interference cancellation (SIC) in virtual MIMO systems. In [18], M. A. Ruder et al. propose strategies using BER as a grouping optimization criterion, where the BER is evaluated after MMSE linear multiuser equalization.

A typical application scenario of user grouping is to combine with frequency resource allocation in the single carrierfrequency division multiple access (SC-FDMA) uplink, where channels are with frequency-selective fading. In order to obtain both the multiuser diversity gain and frequency selective gain, joint resource allocation algorithms with user grouping for SC-FDMA uplink virtual MIMO systems are proposed in [9], [18]. Different from sub-channel allocation for orthogonal frequency division multiplexing access (OFDMA), users can only be assigned to multiple sub-channels that are adjacent to each other in SC-FDMA system [20], [21]. In [18], a low complexity solution combining Hungarian algorithm [22], [23] with binary switching algorithm is proposed for the optimization of joint frequency allocation and user pairing. Since the same number of resources is allocated to each user pair, it is not optimum for system throughput. In [9], a joint user pairing and resource allocation algorithm with flexible 
number of resources and user pairs is proposed to maximize the overall throughput. In [24], in order to obtain a trade-off between throughput and average mean squared error (MSE) performance, the algorithm which includes user grouping and resource allocation in a single cell based on average MSE performance is investigated.

The given algorithms mentioned above focus only on the single-cell scenarios where inter-cell interference (ICI) is neglected. However, as cellular base-stations are more densely deployed, modern cellular networks with small cells are becoming increasingly interference limited. Some research works have been developed on user grouping in multi-cell uplink systems [25]-[31]. In [25], an overview of multi-cell coordinated scheduling and multi-user MIMO techniques in LTE is provided. For the uplink, coordinated reception has been proposed to deal with the ICI, where the coordinated scheduling is performed based on sharing frequency reuse patterns, channel state information (CSI), interference, data and so on. To mitigate ICI, one approach is to schedule multiple users to the same resource blocks (RBs) under the constraint of interference of neighboring cells, where the ICI is treated as noise [26]-[30]. An alternative approach is to effectively exploit rather than control ICI, where joint reception or detection is performed to improve the system performance [31]. A major disadvantage of this scheme is that it needs to share a huge amount of information among coordinated BSs. Most of these studies perform user grouping within each cell and consider the user signals of neighboring cells as interference. In addition, no research specifically focuses on the joint resource allocation algorithm with dynamic singleantenna user grouping in multi-cell SC-FDMA uplink systems. Thus, the joint optimization gains from single-antenna user grouping multiplexing, cell selection and $\mathrm{RB}$ allocation are not fully exploited.

Motivated by the above, we investigate the dynamic singleantenna user grouping across all the coordinated multiple cells in one cluster, to maximize the user grouping multiplexing gain where both the inter-cell and intra-cell interferences are modeled as co-channel transmission of every user group. Simultaneously, a joint resource allocation optimization scheme is designed to achieve maximum $\mathrm{BS}$ selection gain and $\mathrm{RB}$ allocation gain. However, it is hard to solve this binary integer programming problem by using traditional branch-and-bound (BNB) algorithm because the search scale increases dramatically as the numbers of cells, users and RBs increase. To reduce the complexity of the problem solving, we decompose the problem into a series of small scale bipartite matching sub-problems based on partition theory. Hence, an efficient iterative Hungarian algorithm based on user and resource partitions (IHA_URP) is proposed for the solution to the joint resource allocation problem.

Against the existing approaches, our main contributions are as follows:

- To develop dynamic user grouping criteria with BER constraint in multi-cell uplink systems. The criteria are the basis of joint resource allocation algorithm. To ensure the data transmission quality and fully exploit the user grouping and BS selection gains in actual systems, we develop the dynamic grouping criteria using MMSE equalization and adaptive modulation (AM) with BER constraint.

- To formulate a novel joint resource allocation optimization problem which combines multi-cell cooperation, dynamic user grouping and RB allocation for multi-cell SC-FDMA uplink systems. To maximize the cluster-wide performance with multi-cell cooperation, we propose a dynamic user grouping scheme to achieve spatial multiplexing gain by exploiting both intra-cell and inter-cell co-channel users. In the meantime, RBs are allocated orthogonally to different groups to eliminate interference among groups.

- To propose an efficient IHA_URP algorithm for solving the joint resource allocation optimization problem by decomposing it into multiple smaller scale sub-problems. As the numbers of cells, users and RBs increase, the search scale increases dramatically so that the traditional BNB algorithm is unable to solve the problem efficiently. Our proposed new efficient solution algorithm combining iterative Hungarian algorithm with scale compression approach using greedy strategy can significantly reduce the search scale and can also be run in parallel mode for fast processing.

The rest of this paper is organized as follows. Section II presents the multi-cell virtual MIMO system model for the SC-FDMA uplink. In Section III, we first develop a novel multi-cell dynamic user grouping criteria with BER constraint. $\mathrm{We}$, then formulate a joint resource allocation optimization problem for the multi-cell uplink system and propose an efficient IHA_URP algorithm for the solution. Section IV illustrates our simulation results. Section V concludes the paper.

Here are some notations to be used in this paper :

- $\mathbf{I}_{m}, 1_{m \times n}: m \times m$ identity matrix and $m \times n$ matrix with all 1's, respectively.

- $\otimes,(\bullet)^{T},(\bullet)^{H}$ : Kronecker product, transposition, Hermitian, respectively.

- $[\bullet]_{u, u}$ : elements at the $u$-th row and $u$-th column of a matrix.

- $|\bullet|$ : set cardinality.

- $\mathrm{E}(\bullet)$ : expectation operation.

- \lfloor\rfloor : round up operation.

Throughout this paper, all matrices and sets are denoted by capital letters in boldface, and vectors are denoted by lowercase letters in boldface.

\section{SYSTEM MODEL AND PROBLEM DESCRIPTION}

\section{A. Multi-cell Virtual MIMO Model}

Consider a multi-cell uplink virtual MIMO system with $L$ coordinated BSs (i.e. $L$ cells), where each cell contains one BS equipped with $N_{r}$ receiving antennas, $U$ single antenna users. See Figure 1. A similar channel model as given in [32] is considered and can be described as follows: 


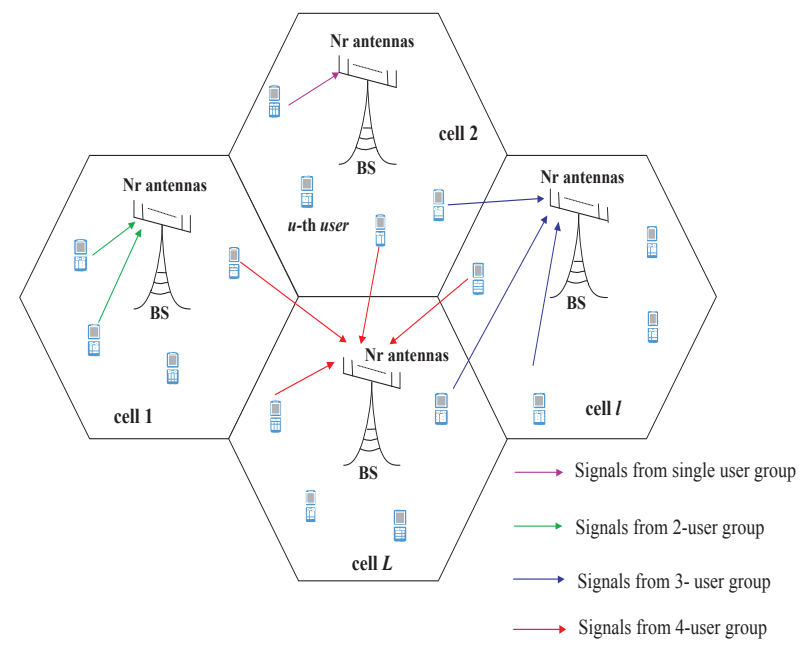

Fig. 1: Illustration of the multi-cell uplink virtual MIMO system with uniform distribution of users $(U)$ and coordinated BSs $(L=4)$

The propagation factor from $u$-th user of the $j$-th cell to the $m$-th antenna of the BS in the $l$-th cell is denoted as $h_{m, l, u, j}=\sqrt{\beta_{m, l, u, j}} \gamma_{m, l, u, j}$, where $\gamma_{m, l, u, j}$ is small scale fading factor, which is independent and identically distributed zero mean, circularly-symmetric complex Gaussian $\mathrm{CN}(0,1)$ random variables, and $\beta_{m, l, u, j}$ is large scale fading coefficient which models the geometric attenuation and shadow fading that are assumed to be constant over a coherence time and known a priori.

In order to increase the spectral efficiency and occupancy level compared with classical FDMA, users in the cluster form virtual MIMO groups and operate over the same timefrequency resource, while different groups occupy orthogonal resources to eliminate the other group interference. In this paper, we select $L$ coordinated cells to form a cluster in which the $L$ cells share the same $N_{R B}$ RBs and the users can pair with each other dynamically across cells. We name such a scheme as the multi-cell cooperation.

There should be a central controller for the implementation of the proposed algorithm in the system considered in this paper. Compared with the single-cell approaches, the proposed multi-cell algorithm requires the exchange of CSI and scheduling information between each cooperative BS and the central processing unit of the cluster, which introduces the backhaul overhead. In this paper, we assume that cooperative BSs are connected to a centralized unit with high-speed backhaul links (e.g. fiber links or microwave) whose bandwidth is sufficient to facilitate the proposed scheduling algorithm.

\section{B. Communication Scheme}

A SC-FDMA uplink system is considered in this paper as shown in Figure 2.

Let $\boldsymbol{\Omega}=\left\{\Omega_{1}, \cdots, \Omega_{g}, \cdots, \Omega_{|\Omega|}\right\}$ denote the set of user groups in the cluster and $\Upsilon_{\Omega_{g}}$ be the set of users in group $\Omega_{g}$. In each consecutive subcarrier chunk, the scheduler chooses $\left|\Upsilon_{\Omega_{g}}\right|$ users among a total of $L U$ users to form a virtual MIMO where $\left|\Upsilon_{\Omega_{g}}\right| \leq N_{r}$ and selects $l$-th BS as data receiver, where $1 \leq l \leq L$. Assume user group $\Omega_{g}$ is scheduled in $M_{g}$ consecutive subcarriers with the first index $p_{g}$, we write the received signal vector of user group $\Omega_{g}$ at $p$-th subcarrier before MIMO detector as

$$
\begin{gathered}
\mathbf{Y}_{p, g, l}=\mathbf{H}_{p, g, l} \mathbf{X}_{p, g, l}+\mathbf{n}_{p, g, l}, \\
p=p_{g}, p_{g}+1, \ldots, p_{g}+M_{g}-1, M_{g}=N_{R B}^{g} N_{s c}^{R B}
\end{gathered}
$$

where $\mathbf{H}_{p, g, l}$ is the $N_{r} \times\left|\boldsymbol{\Upsilon}_{\Omega_{g}}\right|$ virtual MIMO channel matrix, $\mathbf{X}_{p, g, l}$ is the $\left|\mathbf{\Upsilon}_{\Omega_{g}}\right| \times 1$ transmitting signal vector, $\mathbf{n}_{p, g, l}$ is the $N_{r} \times 1$ zero-mean additive white Gaussian noise (AWGN) vector with covariance matrix $E\left\{\mathbf{n}_{p, g, l} \mathbf{n}_{p, g, l}{ }^{H}\right\}=\sigma^{2} \mathbf{I}_{N_{r}}$, $N_{R B}^{g}$ and $N_{\mathrm{sc}}^{R B}$ denote the number of RBs occupied by user group $\Omega_{g}$ and the number of subcarriers in one RB. Perfect power control over user groups is assumed in this paper. So, the total transmitting power of each user group signal vector $\mathbf{X}_{p, g, l}$ is constrained to $E_{s}$, and the transmitting power of each user signal is normalized to $\frac{E_{s}}{\left|\Upsilon_{\Omega_{g}}\right|}$.

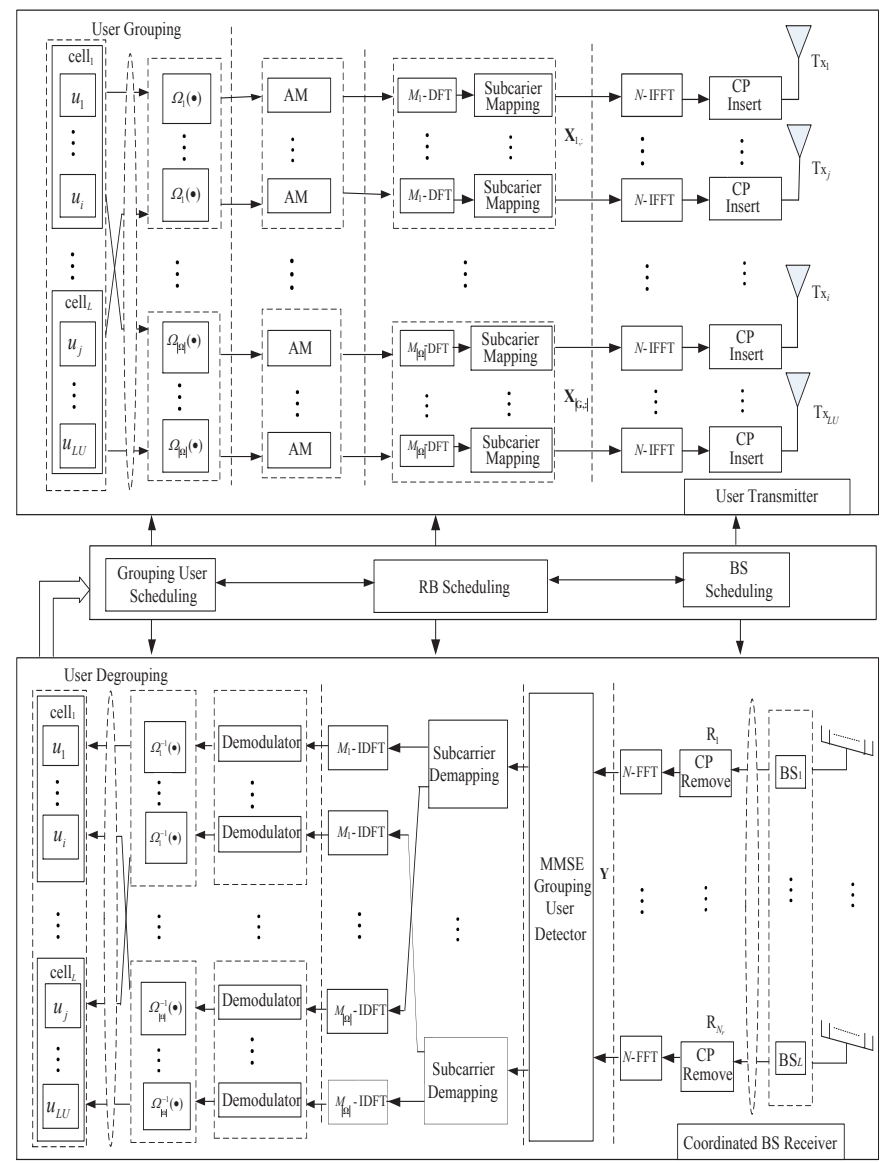

Fig. 2: Block diagram of the multi-cell virtual MIMO for SC-FDMA uplink system

At the BS, MMSE detection is utilized. Then, the detection result can be given as

$$
\widehat{\mathbf{X}_{p, g, l}}=\left(\sigma^{2} \mathbf{I}_{N_{r}}+\mathbf{H}_{p, g, l}{ }^{H} \mathbf{H}_{p, g, l}\right)^{-1} \mathbf{H}_{p, g, l}{ }^{H} \mathbf{Y}_{p, g, l},
$$

where $\sigma^{2}$ denotes the spectral density power of noise. The receiving data of different users is then restored after the subcarrier de-mapping and user de-grouping. 


\section{Problem Clarification}

Three schedulers are involved in the joint resource allocation scheme: user grouping, receiving BS selection and RB assignment. To obtain the maximum system throughput with low complexity, two important problems must be solved.

The first problem is how to design an efficient and new user grouping criterion which achieves good trade-off between throughput and transmission performance. In this paper, we study the criteria for actual system with MMSE equalization and AM techniques where BER constraint is considered.

Another problem is how to effectively combine dynamic user grouping, resource allocation and cell selection together in SC-FDMA uplink. In the next section, we formulate a new combinatorial optimization problem whose objective function is to maximize the system throughput. Three constraints are set in this problem, where the user constraint is that each user can occupy up to one resource pattern, resource constraint is that each RB can only be allocated to one user group and cell constraint is that each user group and RB can only be assigned to one receiving $\mathrm{BS}$.

\section{DYNAMIC USER GROUPING AND JOINT RESOURCE ALLOCATION}

In this section, we first formulate our dynamic user grouping with different number of grouping partners. Then we derive the dynamic user grouping criteria for multi-cell SC-FDMA uplink. Last, we propose the joint user grouping, BS selection and $\mathrm{RB}$ allocation algorithm to optimize the system throughput.

\section{A. Dynamic User Grouping for Multi-cell SC-FDMA Uplink}

The user groups are scheduled on different RBs and receiving BSs in our scheme. Considering the time-frequency correlation, we assume all subcarriers in one RB have the same CSI which can be obtained by taking the average of the CSIs of the subcarriers within the RB. To keep procedures simple, we drop the subscripts of subcarrier/RB and receiving $\mathrm{BS}$ in the description of user grouping. For a multi-cell SCFDMA uplink with $L$ cells and $U$ active users, we write the group set according to the number of users scheduled in one group, that is

$$
\boldsymbol{\Omega}=\left\{\boldsymbol{\Omega}_{1}^{\mathrm{NU}}, \cdots, \boldsymbol{\Omega}_{m}^{\mathrm{NU}}, \cdots, \boldsymbol{\Omega}_{N_{r}}^{\mathrm{NU}}\right\},
$$

where $\boldsymbol{\Omega}_{m}^{\mathrm{NU}}$ denotes $m$-user group subset and superscript "NU" denotes the number of users in each group (NU).

Lemma 1. Let $\boldsymbol{\Omega}_{m}^{\mathrm{NU}}=\left\{\Omega_{m, i}^{\mathrm{NU}}\right\}, 0<i \leq\left|\boldsymbol{\Omega}_{m}^{\mathrm{NU}}\right|$ and $\Omega_{m, i}^{\mathrm{NU}}=\left(u_{1}, u_{2}, \cdots, u_{m}\right)$ denote the $i$-th user group corresponding to user index combination form $\left(u_{1}, u_{2}, \cdots, u_{m}\right)$, where $1 \leq u_{1}<\cdots<u_{m} \leq L U$ and $1 \leq m \leq N_{r}$. Then, the group index $i$ of $\Omega_{m, i}^{\mathrm{NU}}$ can be expressed as

$$
i=\left\{\begin{array}{rl}
\sum_{j=1}^{m-1} \Delta_{j}+\left(u_{m}-u_{m-1}\right), & m>1 \\
u_{1} & , m=1
\end{array},\right.
$$

where $\Delta_{j}=\left(\begin{array}{l}L U-u_{j-1} \\ m-j+1\end{array}\right)-\left(\begin{array}{l}L U-\left(u_{j}-1\right) \\ m-j+1\end{array}\right)$.
Proof:

- $m=1$

It is easy to derive that the group index $i$ can be expressed by user index $u_{1}$.

- $m>1$

For a user group $\Omega_{m, i}^{\mathrm{NU}}=\left(u_{1}, u_{2}, \cdots, u_{m}\right), 1 \leq u_{1}<\cdots<$ $u_{m} \leq L U$, we have $i \in\left[1,\left(\begin{array}{c}L U \\ m\end{array}\right)\right]$.

First, we determine the range of $i$ according to $u_{1}$. The gray segment in the top half of Figure 3 illustrates the range of $i$ when $u_{1}$ is given. Define $\left(\begin{array}{c}L U \\ m\end{array}\right)-\left(\begin{array}{c}L U-\left(u_{1}-1\right) \\ m\end{array}\right)$ as $\Delta_{1}$, then $i \in\left[\Delta_{1}+1, \Delta_{1}+\left(\begin{array}{c}L U-u_{1} \\ m-1\end{array}\right)\right]$, where $\left(\begin{array}{c}L U \\ m\end{array}\right)$ denotes the total number range of $i$ and $\left(\begin{array}{c}L U-\left(u_{1}-1\right) \\ m-1\end{array}\right)$ denotes the number range of $i$ when the first user number is greater than or equals to $u_{1}$.

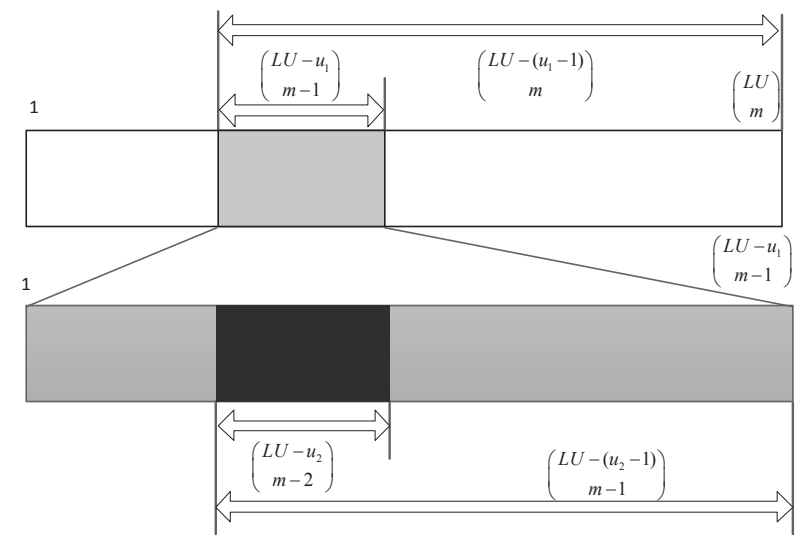

Fig. 3: The range of $i$

Second, we determine the range of $i$ according to $u_{1}$ and $u_{2}$. The black segment in the bottom half of Figure 3 illustrates the range of $i$. Define $\left(\begin{array}{c}L U-u_{1} \\ m-1\end{array}\right)-\left(\begin{array}{c}L U-\left(u_{2}-1\right) \\ m-1\end{array}\right)$ as $\Delta_{2}$, then $i \in\left[\Delta_{1}+\Delta_{2}+1, \Delta_{1}+\Delta_{2}+\left(\begin{array}{c}L U-u_{2} \\ m-2\end{array}\right)\right]$, where $\left(\begin{array}{c}L U-u_{2} \\ m-2\end{array}\right)$ denotes the number range of $i$ when the first and second user number are greater than or equal to $u_{1}$ and $u_{2}$ respectively.

Then, repeat the similar step until reaching the condition of $u_{m-1}$. Correspondingly, define $\left(\begin{array}{c}L U-u_{j-1} \\ m-j+1\end{array}\right)-\left(\begin{array}{c}L U-\left(u_{j}-1\right) \\ m-j+1\end{array}\right) \quad$ as $\quad \Delta_{j}, \quad$ then $i \in\left[\sum_{j=1}^{m-1} \Delta_{j}+1, \sum_{j=1}^{m-1} \Delta_{j}+\left(\begin{array}{c}L U-u_{m-1} \\ 1\end{array}\right)\right]$.

Finally, when given $u_{1}, u_{2}, u_{3}, \cdots, u_{m}$, we can eventually obtain Eq. (4):

$$
i=\sum_{j=1}^{m-1} \Delta_{j}+\left(u_{m}-u_{m-1}\right), m>1 .
$$


Example 1. We assume that $L=1, U=20, m=4$ and the receiving antennas at $\mathrm{BS}$ is $N_{r}=4$. Let $\mathbf{B}^{m}$ be $m$-user grouping matrix. Taking 4-user grouping as an example, we can write $\mathbf{B}^{4}$ as follows:

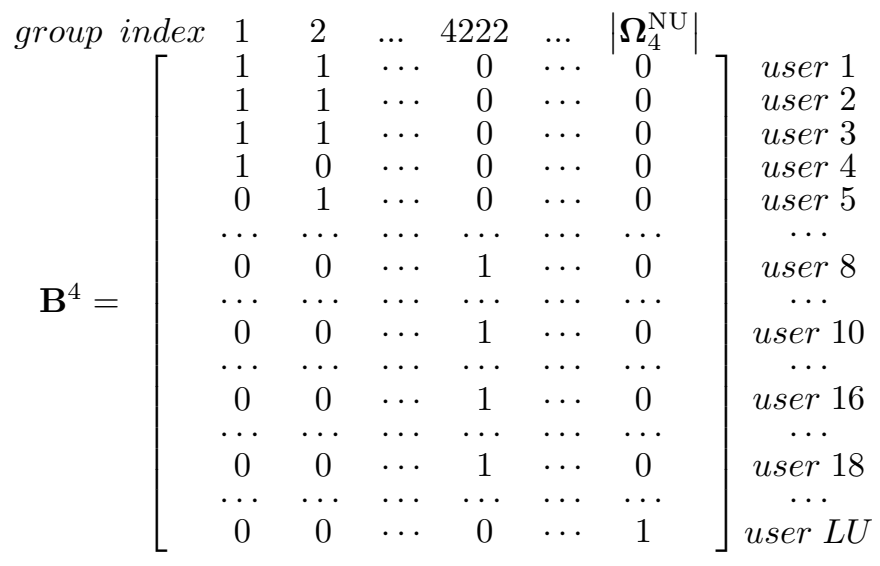

where each element indicates whether the user is involved in the group or not. According to Eq. (4), if group $\Omega_{m, i}^{\mathrm{NU}}=(8,10,16,18)$, we can get the index $i$ of $\Omega_{m, i}^{\mathrm{NU}}$ in set $\boldsymbol{\Omega}_{m}^{\mathrm{NU}}$ equals 4222 , which is demonstrated in the 4222 -th column of $\mathbf{B}^{4}$.

As the number of all user groups in set $\boldsymbol{\Omega}$ is $|\boldsymbol{\Omega}|=\sum_{m=1}^{N_{r}}\left|\boldsymbol{\Omega}_{m}^{\mathrm{NU}}\right|=\sum_{m=1}^{N_{r}}\left(\begin{array}{c}L U \\ m\end{array}\right)$, based on Lemma 1, we can write the index of user group $\Omega_{g}$ in set $\Omega$ as

$g=\left\{\begin{array}{c}\sum_{n=1}^{m-1}\left(\begin{array}{c}L U \\ n\end{array}\right)+\sum_{j=1}^{m-1} \Delta_{j}+\left(u_{m}-u_{m-1}\right), m>1 \\ u_{m}\end{array}\right.$,

where set $\boldsymbol{\Omega}=\left\{\Omega_{1}, \cdots, \Omega_{g}, \cdots, \Omega_{|\boldsymbol{\Omega}|}\right\}$ and user group $\Omega_{g}=$ $\left(u_{1}, u_{2}, \cdots, u_{m}\right)$.

For example, the index of $\Omega_{g}=\Omega_{m, i}^{\mathrm{NU}}=(8,10,16,18)$ in set $\Omega$ is $g=\sum_{n=1}^{m-1}\left(\begin{array}{c}L U \\ n\end{array}\right)+\sum_{j=1}^{m-1} \Delta_{j}+\left(u_{m}-u_{m-1}\right)=5572$.

Then, the maximum capacity of virtual MIMO from user group $\Omega_{g}$ to $l$-th receiving BS can be expressed as

$$
\Psi_{g, l}=\log _{2} \operatorname{det}\left(\mathbf{I}_{N_{r}}+\frac{E_{s}}{\left|\boldsymbol{\Upsilon}_{\Omega_{g}}\right| \sigma^{2}} \mathbf{H}_{g, l} \mathbf{H}_{g, l}{ }^{H}\right) .
$$

To achieve the maximum system capacity, the receiving BS selection and dynamic user grouping criterion can be written as

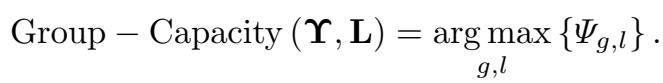

The criterion (7) shows the ideal system throughput for grouped users, however, it does not give the user grouping rule with MIMO equalization and demodulation techniques. In this paper, we consider the MMSE equalization and AM with BER constraint for virtual MIMO systems. From Eq. (2), we get the receiving SNR of user $u$ at $l$-th receiving BS after MIMO equalization as [9]

$$
S N R_{u, l}=\frac{E_{s}}{\sigma^{2}\left[\left(\mathbf{H}_{g, l}{ }^{H} \mathbf{H}_{g, l}+\frac{\sigma^{2}}{E_{s}} \mathbf{I}_{\left|\boldsymbol{\Upsilon}_{\Omega_{g}}\right|}\right)^{-1}\right]_{u, u}}-1 .
$$

Then, the capacity using post-processing SNR can be expressed as

$$
\Psi_{g, l}{ }^{\text {Post }}=\sum_{u \in \Upsilon_{\Omega_{g}}} \log _{2}\left(1+S N R_{u, l}\right) .
$$

The capacity in Eq. (9) is the upper bound for ideal transmissions after MMSE equalization. When we consider the practical techniques, such as AM techniques, the transmission rate is decided by the SNR and the BER through the use of modulation techniques. Specially, we consider a variable rate modulation using $\mathrm{M}$-ary quadrature amplitude modulation (MQAM) in this work. In [33], the BER for an AWGN channel with MQAM and ideal coherent detection is bounded by

$$
\mathrm{BER}^{\varphi} \leq 0.2 e^{\frac{-1.5 S N R}{2 \varphi-1}},
$$

where $2^{\varphi}$ is the modulation order. Then, the maximum spectral efficiency of user $u$ after AM techniques is

$$
\varphi_{u, l}=\left\lfloor\log _{2}\left(1-\frac{1.5 S N R_{u, l}}{\ln \left(5 \mathrm{BER}^{\text {target }}\right)}\right)\right\rfloor,
$$

where BER ${ }^{\text {target }}$ is the upper bound BER constraint for signal transmission. So the transmission efficiency of the virtual MIMO is

$$
R_{g, l}=\sum_{u \in \Upsilon_{\Omega_{g}}} \varphi_{u, l} .
$$

Then, we get the user grouping criterion based on MMSE equalization and $\mathrm{AM}$ techniques as

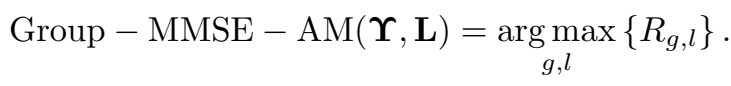

In addition, when user fairness needs to be taken into account, the transmission efficiency of each user should be weighted by a fairness factor. An effective approach to get the fairness factor is using proportional scheduling. For criterion (13), the weighted factor for spectral efficiency of user $u$, i.e. $\varphi_{u, l}$, at $n$-th time slot can be calculated based on smoothing window method as follows [34]:

$$
w_{u, l} \mathrm{FP}(n)=\frac{1}{\bar{\varphi}_{u, l}(n)},
$$

where

$\bar{\varphi}_{u, l}(n)= \begin{cases}\left(1-\frac{1}{T_{\mathrm{W}}}\right) \bar{\varphi}_{u^{\prime}, l}(n-1)+\frac{1}{T_{\mathrm{W}}} \varphi_{u^{\prime}, l}, & u=u^{\prime} \\ \left(1-\frac{1}{T_{\mathrm{W}}}\right) \bar{\varphi}_{u, l}(n-1), & \text { others }\end{cases}$

and $T_{\mathrm{W}}$ is smoothing window length.

\section{B. Joint User Grouping and Resource Allocation in Multi-cell SC-FDMA Uplink}

For multi-cell SC-FDMA uplink system, adjacent timefrequency RBs which can only belong to one receiving BS should be assigned to one user group. Assume that $N_{R B}$ consecutive RBs are available to be allocated to users, we can determine the allocation pattern number $J=\frac{N_{R B}\left(N_{R B}+1\right)}{2}+1$. Hence the resource pattern matrix $\mathbf{T}$ is expressed as follows [20]: 


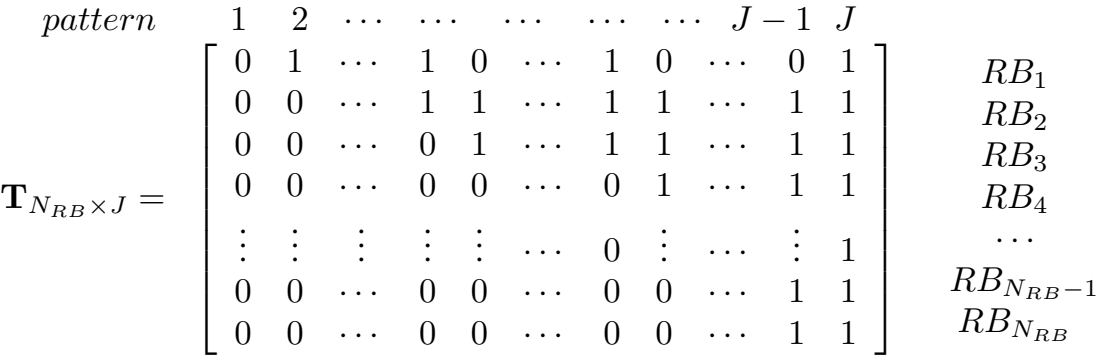

Based on the idea of criteria for receiving BS selection, user grouping and RBs allocation, we first calculate the RB resource metric matrix for user groups at $l$-th receiving BS as follows:

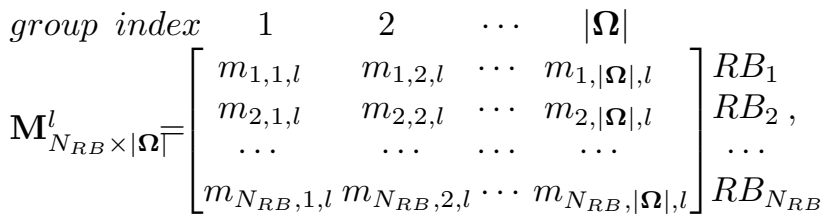

where the elements $\left\{m_{j, g, l}\right\}$ are calculated according to Eq.(12), i.e. $m_{j, g, l}=R_{j, g, l}$.

To achieve BS selection gain in one cluster, we derive the metric for all RBs, user groups and receiving BSs as follows:

$\mathbf{M}_{N_{R B},|\boldsymbol{\Omega}| L}=\left[\mathbf{M}_{N_{R B} \times|\boldsymbol{\Omega}|}^{1}, \cdots, \mathbf{M}_{N_{R B} \times|\boldsymbol{\Omega}|}^{l}, \cdots, \mathbf{M}_{N_{R B} \times|\boldsymbol{\Omega}|}^{L}\right]$.

From resource pattern (15), we express the transmission rate for $g$-th user group to $l$-th receiving BSs at $j$-th resource pattern as

$$
\eta_{j, g, l}=\operatorname{sum}\left(\mathbf{M}_{N_{R B} \times|\boldsymbol{\Omega}|}^{l}(:, g) \cdot * \mathbf{T}(:, j)\right) .
$$

Define $|\boldsymbol{\Omega}| J L \times 1$ resource allocation vector $\boldsymbol{\delta}$ and $|\boldsymbol{\Omega}| J L \times$ 1 rate vector of user groups $\boldsymbol{\eta}$ as

$$
\begin{gathered}
\boldsymbol{\delta}=\left[\delta_{1,1,1}, \cdots, \delta_{J, 1,1}, \cdots, \delta_{j, g, 1}, \cdots, \delta_{J,|\Omega|, 1}, \cdots, \delta_{j, g, l},\right. \\
\text { and } \\
\left.\quad \cdots, \delta_{1,1, L}, \cdots, \delta_{J, 1, L}, \cdots, \delta_{j, g, L}, \cdots, \delta_{J,|\Omega|, L}\right]^{T} \\
\boldsymbol{\eta}=\left[\eta_{1,1,1}, \cdots, \eta_{J, 1,1}, \cdots, \eta_{j, g, 1}, \cdots, \eta_{J,|\Omega|, 1}, \cdots, \eta_{j, g, l},\right. \\
\left.\quad \cdots, \eta_{1,1, L}, \cdots, \eta_{J, 1, L}, \cdots, \eta_{j, g, L}, \cdots, \eta_{J,|\Omega|, L}\right]^{T}
\end{gathered}
$$

where $\delta_{j, g, l}$ is the assignment index indicating $g$-th user group occupying $j$-th RB pattern in $l$-th cell and $\delta_{j, g, l}=\{0,1\}, j=$ $1, \cdots, J ; g=1, \cdots|\Omega| ; l=1, \cdots L$.

Then, we write the optimization problem of the joint resource allocation as M_JRA $(\boldsymbol{\Omega}, \mathbf{T}, L)$

$$
\arg \max _{\boldsymbol{\delta}}\left\{\boldsymbol{\eta}^{T} \boldsymbol{\delta}\right\}
$$

subject to

$$
\begin{gathered}
\mathrm{AC} 1: \boldsymbol{A}_{\mathbf{1}} \boldsymbol{\delta} \leq 1_{N_{R B} \times 1} \\
\mathrm{AC} 2: \boldsymbol{A}_{\mathbf{2}} \boldsymbol{\delta} \leq 1_{U \times 1} \\
\mathrm{AC} 3: \boldsymbol{A}_{3} \boldsymbol{\delta} \leq 1_{L \times 1} \\
\mathrm{AC} 4: \mathrm{BER}^{\varphi} \leq \mathrm{BER}^{\text {target }} \\
\mathrm{AC} 5: \delta_{j, g, l}=\{0,1\}, \\
j=1, \cdots, J ; \mathrm{g}=1, \cdots|\boldsymbol{\Omega}| ; l=1, \cdots L
\end{gathered}
$$

where $\mathbf{A}_{\mathbf{1}}=1_{1 \times L} \otimes\left(\mathbf{T} \otimes 1_{1 \times|\Omega|}\right), \quad \mathbf{A}_{\mathbf{2}}=1_{1 \times L} \otimes$ $\left(1_{1 \times J} \otimes \mathbf{B}\right), \quad \mathbf{A}_{3}=\left(1_{1 \times J} \otimes 1_{1 \times|\Omega|}\right) \otimes \mathbf{I}_{L}$, and $\mathbf{B}=$
$\left[\mathbf{B}^{1}, \quad \mathbf{B}^{2}, \cdots, \quad \mathbf{B}^{m}, \cdots, \quad \mathbf{B}^{N_{r}}\right]$ is the user grouping matrix.

The aim of (19) is to maximize the total throughput. The constraint $\mathrm{AC} 1$ is to ensure that each $\mathrm{RB}$ can only be allocated to one user group, AC2 is to ensure that each user can occupy one resource pattern at most, AC3 is to ensure that each user group and $\mathrm{RB}$ can only belong to one receiving $\mathrm{BS}$ and $\mathrm{AC} 4$ is the BER constraint.

\section{Solution Algorithm to the Joint Resource Allocation Prob- lem}

The optimization problem (19) is a typical binary integer programming problem. So it can be converted to Office Assignment Problem (OAP) [35] and use a linear programming (LP)-based BNB algorithm [36] to solve the problem.

However, BNB algorithm is too complex for a practical implementation especially when the number of cells, users and RBs becomes large. In order to reduce the complexity, we decompose the problem (19) into a series of lower degree dimension sub-problems which can be modeled as bipartite graphs. Based on the consideration of constraints (19a) and (19b), we regroup the elements in set $\Omega$ to create the complete user group set $\boldsymbol{\Omega}^{\mathrm{CUG}}=\left\{\boldsymbol{\Omega}_{i}^{\mathrm{CUG}}\right\}$ so that each subset $\boldsymbol{\Omega}_{i}^{\mathrm{CUG}}$ contains the groups with all users but has no duplicate users. Similarly, we create the complete RB pattern set $\mathbf{T}^{\mathrm{CRP}}=$ $\left\{\mathbf{T}_{j}^{C R P}\right\}$ so that each subset $\mathbf{T}_{j}^{C R P}$ contains the RB pattern with all RBs but has no duplicate RBs.

1) Construction of the Complete RB Pattern Set $\mathbf{T}^{\mathrm{CRP}}$ :

Lemma 2. If there are $N_{R B}$ RBs available to be allocated, the size of the RB pattern set $\mathbf{T}^{\mathrm{CRP}}$ will be $2^{N_{R B}-1}$.

Proof: Considering a $N_{R B}$-RB sequence with the order of $R B_{1}, R B_{2}, \cdots, R B_{N_{R B}}$, we insert a underline between each of $\mathrm{RBs}$ to construct a new sequence

$$
\omega=\left\{R B_{1}, \ldots, R B_{2}, \ldots, \cdots, R B_{N_{R B}}\right\} .
$$

Only 0 and 1 can be put on these underlines. Putting 0 on the underline between $R B_{i}$ and $R B_{i+1}$ means that $R B_{i}$ and $R B_{i+1}$ are assigned to the same RB pattern and vice versa. Through changing the values put on these underlines, we can obtain all pattern subset $\mathbf{T}_{j}^{\mathrm{CRP}}$ and derive the size of the RB pattern set $\left|\mathbf{T}^{\mathrm{CRP}}\right|=2^{N_{R B}-1}$ by multiplication principle.

- Generating The Indexes of Elements in $\mathrm{T}^{\mathrm{CRP}}$ :

Define $j$ to be the index of subset $\mathbf{T}_{j}^{C R P}$ in $\mathbf{T}^{\mathrm{CRP}}$, then, $j$ equals the value of the binary sequence inserted into sequence $\omega$ corresponding to $\mathbf{T}_{j}^{\mathrm{CRP}}$. 
Example 2. Assume there are $N_{R B}=6 \mathrm{RBs}$ in the system, which are $R B_{1}, R B_{2}, \cdots, R B_{6}$. Given the inserted binary sequence $(1,1,0,0,1)$, the sequence $\omega$ is shown as follows:

$\omega=\left\{R B_{1}, \underline{1}, R B_{2}, \underline{1}, R B_{3}, \underline{0}, R B_{4}, \underline{0}, R B_{5}, \underline{1}, R B_{6}\right\}$.

It means that all $\mathrm{RBs}$ are divided into $4 \mathrm{RB}$ patterns to form a complete $\mathrm{RB}$ pattern subset $\mathbf{T}_{25}^{\mathrm{CRP}}=$ $\left\{\left(R B_{1}\right),\left(R B_{2}\right),\left(R B_{3}, R B_{4}, R B_{5}\right),\left(R B_{6}\right)\right\}$ whose index is 25 in $\mathbf{T}^{\mathrm{CRP}}$.

2) Construction of the Complete User Group Set $\Omega^{\mathrm{CUG}}$ : With the integer partition of $L U$ users, we can know the $L U$ users can be divided into how many groups and each group contains how many users. Then, by the operation of user permutations and combinations based on the partition result, we can obtain all the complete user subsets.

- Generation Process of Complete User Group Set $\Omega^{\mathrm{CUG}}$ :

We assume that the total user number in one cluster is $L U$ and the receiving antennas number at each BS is $N_{r}$.

Step1. Generating the integer partition of $L U$

Call the Procedure \#1 shown in APPENDIX A to complete the integer partition operation of $L U$ and obtain the partition matrix $\mathbf{Q}$ whose sum of each row vector equals $L U$. Here, the integer partition operation of $L U$ means listing all possible values of $n_{1}, n_{2}, \cdots, n_{L U}$, which meet the requirement of the following equation:

$$
n_{1}+n_{2}+\cdots+n_{i}+\cdots+n_{L U}=L U,
$$

where $0 \leq n_{i} \leq n_{i+1} \leq N_{r}(1 \leq i \leq L U-1)$.

Step2. Generating the user group set $\Omega^{\mathrm{CUG}}$ based on the integer partition of $L U$

Let $\hat{\mathbf{q}}_{i}$ be the $i$-th row vector of $\mathbf{Q}$. Then, obtain set $\mathbf{q}_{i}=$ $\left\{q_{i, 1}, q_{i, 2}, \cdots, q_{i, W}\right\}$ by deleting the 0 elements in $\hat{\mathbf{q}}_{i}$, where $0<q_{i, 1} \leq q_{i, 2} \leq \cdots \leq q_{i, W} \leq L U, W \leq L U$. Perform the similar operations until each row vector of $\mathbf{Q}$ is traversed.

Select $q_{i, 1}$ users from $L U$ users, and call the Procedure \#2 shown in APPENDIX A to create user group $\boldsymbol{\Omega}_{\mathbf{q}_{i, 1}}^{\mathrm{NU}}$, and then select $q_{i, 2}$ users from the rest of $L U-q_{i, 1}$ users to create user group $\boldsymbol{\Omega}_{\mathbf{q}_{i, 2}}^{\mathrm{NU}}$, repeat the similar operations until user group $\Omega_{\mathbf{q}_{i, W}}^{\mathrm{NU}}$ is obtained.

Let $\boldsymbol{\Omega}_{\mathbf{q}_{i}}^{\mathrm{CUG}}=\left\{\boldsymbol{\Omega}_{\mathbf{q}_{i, 1}}^{\mathrm{NU}}, \boldsymbol{\Omega}_{\mathbf{q}_{i, 2}}^{\mathrm{NU}}, \cdots, \boldsymbol{\Omega}_{\mathbf{q}_{i, W}}^{\mathrm{NU}}\right\}$. Then put all complete user group subsets into set $\Omega^{\mathrm{CUG}}$ in the order of $i$, and finally obtain the complete user group set $\Omega^{\mathrm{CUG}}$.

In addition, according to the analyses above, we can derive the size of $\Omega_{\mathbf{q}_{i}}^{\mathrm{CUG}}$ as follows:

$\Xi_{i}=\frac{\left(\begin{array}{c}L U \\ q_{i, W}\end{array}\right)\left(\begin{array}{c}L U-q_{i, W} \\ q_{i, W-1}\end{array}\right) \cdots\left(\begin{array}{c}L U-\sum_{w=2}^{W} q_{i, w} \\ q_{i, 1}\end{array}\right)}{\xi_{1} ! \xi_{2} ! \cdots \xi_{r} ! \cdots \xi_{N_{r}} !}$,

where $\xi_{r}, 1 \leq r \leq N_{r}$ is the number of elements in $\mathbf{q}_{i}=$ $\left\{q_{i, 1}, q_{i, 2}, \cdots, q_{i, W}\right\}$, which is equal to $r$.

Example 3. Let $N_{r}=4, L U=5$, and the user set is $\left\{u_{1}, u_{2}, u_{3}, u_{4}, u_{5}\right\}$.

Step1:
From Procedure \#1, we can derive that the partition number of $L U$ is 6 and all the partitions are $(1,1,1,1,1),(1,1,1,2,0)$, $(1,1,3,0,0),(1,2,2,0,0),(1,4,0,0,0),(2,3,0,0,0)$. Then, the partition matrix $\mathbf{Q}$ can be expressed as follows:

$$
\mathbf{Q}=\left[\begin{array}{lllll}
1 & 1 & 1 & 1 & 1 \\
1 & 1 & 1 & 2 & 0 \\
1 & 1 & 3 & 0 & 0 \\
1 & 2 & 2 & 0 & 0 \\
1 & 4 & 0 & 0 & 0 \\
2 & 3 & 0 & 0 & 0
\end{array}\right]
$$

where $\mathbf{q}_{2}=(1,1,1,2)$ means that 5 users are divided into 4 groups and each group contains 1 user, 1 user, 1 user and 2 users respectively.

Step2:

Take $\mathbf{q}_{2}=(1,1,1,2)$ as an example, we can get the size of $\Omega_{\mathbf{q}_{2}}^{\mathrm{CUG}}$ as

$\Xi_{2}=\frac{\left(\begin{array}{l}5 \\ 1\end{array}\right)\left(\begin{array}{c}5-1 \\ 1\end{array}\right)\left(\begin{array}{c}5-1-1 \\ 1\end{array}\right)\left(\begin{array}{c}5-1-1-1 \\ 2\end{array}\right)}{3 ! 1 !}=10$.

The complete user group subsets in $\Omega_{\mathbf{q}_{2}}^{\mathrm{CUG}}$ corresponding to $\mathbf{q}_{2}$ is

$$
\mathbf{\Omega}_{\mathbf{q}_{\mathbf{2}}}^{\mathrm{CU}}=\left\{\begin{array}{llll}
\boldsymbol{\Omega}_{\mathbf{q}_{\mathbf{2}, \mathbf{1}}}^{\mathrm{NU}} & \mathbf{\Omega}_{\mathbf{q}_{\mathbf{2}, \mathbf{2}}}^{\mathrm{NU}} & \boldsymbol{\Omega}_{\mathbf{\mathbf { q } _ { 2 } , \mathbf { 3 }}}^{\mathrm{NU}} & \boldsymbol{\Omega}_{\mathbf{q}_{\mathbf{2}, \mathbf{4}}}^{\mathrm{NU}} \\
\left\{\left(u_{1}\right)\right. & \left(u_{2}\right) & \left(u_{3}\right) & \left.\left(u_{4}, u_{5}\right)\right\} \\
\left\{\left(u_{1}\right)\right. & \left(u_{2}\right) & \left(u_{4}\right) & \left.\left(u_{3}, u_{5}\right)\right\} \\
\left\{\left(u_{1}\right)\right. & \left(u_{2}\right) & \left(u_{5}\right) & \left.\left(u_{3}, u_{4}\right)\right\} \\
\left\{\left(u_{1}\right)\right. & \left(u_{3}\right) & \left(u_{4}\right) & \left.\left(u_{2}, u_{5}\right)\right\} \\
\left\{\left(u_{1}\right)\right. & \left(u_{3}\right) & \left(u_{5}\right) & \left.\left(u_{2}, u_{4}\right)\right\} \\
\left\{\left(u_{1}\right)\right. & \left(u_{4}\right) & \left(u_{5}\right) & \left.\left(u_{2}, u_{3}\right)\right\} \\
\left\{\left(u_{2}\right)\right. & \left(u_{3}\right) & \left(u_{4}\right) & \left.\left(u_{1}, u_{5}\right)\right\} \\
\left\{\left(u_{2}\right)\right. & \left(u_{3}\right) & \left(u_{5}\right) & \left.\left(u_{1}, u_{4}\right)\right\} \\
\left\{\left(u_{2}\right)\right. & \left(u_{4}\right) & \left(u_{5}\right) & \left.\left(u_{1}, u_{3}\right)\right\} \\
\left\{\left(u_{3}\right)\right. & \left(u_{4}\right) & \left(u_{5}\right) & \left.\left(u_{1}, u_{2}\right)\right\}
\end{array}\right\} .
$$

Add elements of $\Omega_{\mathbf{q}_{2}}^{\mathrm{CUG}}$ into the complete user group set $\Omega^{\mathrm{CUG}}$.

- Size Analysis of Complete User Group Set $\Omega^{\mathrm{CUG}}$ :

The size of complete user group set $\Omega^{\mathrm{CUG}}$ mainly depends on the partitions of $L U$ users. In the following, we first investigate the partition number of $L U$ users with the group user number limit $N_{r}$, then, discuss the size of complete user group set $\Omega^{\mathrm{CUG}}$

Lemma 3. Let $P \leq N_{r}(L U)$ be the partition number of $L U$ with no parts greater than $N_{r}$, we have the following theorem:

$$
P^{\leq N_{r}}(L U)=\sum_{m=0}^{L U} P^{\leq N_{r}-1}(m) \times b_{L U-m}, P^{\leq N_{r}-1}(0)=1 \text {, }
$$

where $b_{m}=\left\{\begin{array}{l}1, m=i * L U, i=0,1, \cdots \\ 0, \text { others }\end{array}\right.$ is the coefficient of $t^{m}$ in the Taylor's expansion of $\frac{1}{\left(1-t^{L U}\right)}$.

Proof: Let $A\left(L U, N_{r}-1\right)$ be the number of positive solutions of Diophantine equation $\sum_{i=1}^{N_{r}-1} i x_{i}=L U$ and 
$p^{\left(\leq N_{r}-1\right)}(t)=\frac{1}{(1-t)\left(1-t^{2}\right) \cdots\left(1-t^{N_{r}-1}\right)}$ be the generating function. Here $A\left(L U, N_{r}-1\right)$ is the coefficient of $t^{L U}$ in the $p^{\left(\leq N_{r}-1\right)}(t)$.

Generating function $p^{\left(\leq N_{r}-1\right)}(t)$ can be expressed as

$$
\begin{aligned}
p^{\left(\leq N_{r}-1\right)}(t) & =\frac{1}{(1-t)\left(1-t^{2}\right) \cdots\left(1-t^{N_{r}-1}\right)} \\
& =\sum_{i=0}^{\infty} A\left(i, N_{r}-1\right) t^{i}
\end{aligned}
$$

The Taylor's expansion of $\frac{1}{\left(1-t^{L U}\right)}$ is $\frac{1}{\left(1-t^{L U}\right)}=\sum_{j=0}^{\infty} b_{j} t^{j}$, so the generating function $p^{\left(\leq N_{r}\right)}(t)$ can be expressed as:

$$
\begin{aligned}
p^{\left(\leq N_{r}\right)}(t) & =\frac{1}{(1-t)\left(1-t^{2}\right) \cdots\left(1-t^{N_{r}-1}\right)} \times \frac{1}{\left(1-t^{N_{r}}\right)} \\
& =\sum_{i=0}^{\infty} A\left(i, N_{r}-1\right) t^{i} \times \sum_{j=0}^{\infty} b_{j} t^{j} \\
& =\sum_{s=0}^{\infty}\left[\sum_{m=0}^{s} A\left(m, N_{r}-1\right) \times b_{s-m}\right] t^{s}
\end{aligned}
$$

We select the coefficient of $t^{L U}$, then the following conclusion can be obtained:

$$
A\left(L U, N_{r}\right)=\sum_{m=0}^{L U} A\left(m, N_{r}-1\right) \times b_{L U-m} .
$$

Due to $P \leq\left(N_{r}\right)(L U)=A\left(L U, N_{r}\right)$, we can know that:

$$
\begin{aligned}
P^{\leq\left(N_{r}\right)}(L U) & =A\left(L U, N_{r}\right) \\
& =\sum_{m=0}^{L U} A\left(m, N_{r}-1\right) \times b_{L U-m} \\
& =\sum_{m=0}^{L U} P^{\leq\left(N_{r}-1\right)}(m) \times b_{L U-m}
\end{aligned}
$$

It cannot obtain the size of complete user group set $\Omega^{\mathrm{CUG}}$ from $P \leq\left(N_{r}\right)(L U)$ directly. However, we can calculate the size $\left|\Omega^{\mathrm{CUG}}\right|$ using Eq. (21) after the partition procedure of user $L U$.

Lemma 4. The size of complete user group set $\Omega^{\mathrm{CUG}}$ is

$$
\begin{aligned}
& \quad \Omega^{\mathrm{CUG}} \mid=\sum_{i} \Xi_{i} \\
& =\sum_{i} \frac{\left(\begin{array}{c}
L U \\
q_{i, W}
\end{array}\right)\left(\begin{array}{c}
L U-q_{i, W} \\
q_{i, W-1}
\end{array}\right) \cdots\left(\begin{array}{c}
L U-\sum_{w=2}^{W} q_{i, w} \\
q_{i, 1}
\end{array}\right)}{\xi_{1} ! \xi_{2} ! \cdots \xi_{r} ! \cdots \xi_{N_{r}} !}
\end{aligned}
$$

where $0<q_{i, 1} \leq q_{i, 2} \leq \cdots \leq q_{i, W} \leq L U, W \leq L U$.

Proof: The proof can be easily extended from combination and multiplication principles by considering all partition results. We omit the details due to page limit.

3) Algorithm for the Solution of Joint Resource Allocation Problem: After the generation of complete RB pattern set and complete user group set, iterative Hungarian algorithm can be used to obtain the resource allocation. However, according to Lemma 4 , the size of complete user group set $\left|\Omega^{\mathrm{CUG}}\right|$ grows rapidly with the increase of user and $\mathrm{RB}$ numbers so that the computational complexity is still unbearable. Actually, when given a complete RB pattern subset, only one complete user group subset which belongs to a user partition can be selected as the best match. So, we can compress the complete user group set according to the user partition results before joint resource allocation using greedy strategy. Inspired by these ideas, we present an efficient sub-optimal IHA_URP algorithm to solve problem (19) with the following two phases:

i. Initial phase: integer partitions of users and RBs

From section III. C. 1) and 2), we can obtain integer partitions of $L U$ and $N_{R B}$. Since RBs must be adjacent in one pattern, complete RB pattern set $\mathrm{T}^{\mathrm{CRP}}$ can be obtained as well.

ii. Running phase: complete user group set compression and joint resource allocation

First, compress the size of $\Omega^{\mathrm{CUG}}$ according to the user partition results by greedy algorithm. When given a complete RB pattern subset $\mathbf{T}_{j}^{C R P}$, we can get $P \leq N_{r}(L U)$ best matched complete user group subsets based on partition results.

Then, perform joint resource allocation. Select the complete user group subset and complete RB group subset with maximal sum-rate from the results of previous process.

The detailed workflow is described in the following.

- Generation of the Best Matched Complete User Group Subset from a User Partition:

Given a partition $\mathbf{q}_{i}=\left\{q_{i, 1}, \cdots, q_{i, W}\right\}$ and a complete RB pattern subset $\mathbf{T}_{j}^{\mathrm{CRP}}=\left\{\mathbf{T}_{j, 1}^{\mathrm{CRP}}, \cdots, \mathbf{T}_{j, V}^{\mathrm{CRP}}\right\}$, we can generate the best matched complete user group subset using Hungarian algorithm with greedy strategy.

Step1:

Based on Lemma 1, the performance measure matrix of $\mathbf{T}_{j}^{\mathrm{CRP}}$ and $\boldsymbol{\Omega}_{\mathbf{q}_{i}}^{\mathrm{NU}}$ for cell $l$ can be constructed as follows:

$$
\begin{aligned}
& \text { partition } \\
& \begin{array}{cccccc}
\text { group } & \boldsymbol{\Omega}_{q_{i, 1}}^{\mathrm{NU}} & \ldots & \boldsymbol{\Omega}_{q_{i, w}}^{\mathrm{NU}} & \ldots & \boldsymbol{\Omega}_{q_{i, W}}^{\mathrm{NU}}
\end{array} \\
& \widehat{\boldsymbol{\eta}}_{V \times W, l}=\left[\begin{array}{cccccc}
\widehat{\eta}_{1,1, l} & \ldots & \widehat{\eta}_{1, w, l} & \ldots & \widehat{\eta}_{1, W, l} \\
\cdots & \ldots & \ldots & \ldots & \ldots & \mathbf{T}_{j, 1}^{\mathrm{CRP}} \\
\ldots \\
\widehat{\eta}_{v, 1, l} & \ldots & \widehat{\eta}_{v, w, l} & \ldots & \widehat{\eta}_{v, W, l} \\
\cdots & \ldots & \ldots & \ldots & \ldots & \mathbf{T}_{j, v}^{\mathrm{CRP}} \\
\widehat{\eta}_{V, 1, l} & \cdots & \widehat{\eta}_{V, w, l} & \ldots & \widehat{\eta}_{V, W, l}
\end{array}\right] \mathbf{T}_{j, V}^{\mathrm{CRP}}
\end{aligned}
$$

where $\widehat{\eta}_{v, w, l}=\max _{g \in \boldsymbol{\Omega}_{q_{i, w}^{\mathrm{NU}}}}\left\{\eta_{v, g, l}\right\}$, the corresponding group index $\hat{g}=\underset{g \in \boldsymbol{\Omega}_{q_{i, w} \mathrm{NU}}}{\arg \max }\left\{\eta_{v, g, l}\right\}$ and $1 \leq l \leq L$.

Then, the performance measure matrix of $\mathbf{T}_{j}^{\mathrm{CRP}}$ and $\boldsymbol{\Omega}_{\mathbf{q}_{i}}^{\mathrm{NU}}$ for all cells in one cluster is

$$
\widehat{\boldsymbol{\eta}}=\left[\widehat{\boldsymbol{\eta}}_{V \times W, 1}, \cdots, \widehat{\boldsymbol{\eta}}_{V \times W, l}, \cdots, \widehat{\boldsymbol{\eta}}_{V \times W, L}\right] .
$$

Step2:

Find the best match between resource pattern and user group by the operation $\left(v^{*}, w^{*}, l^{*}, g^{*}\right)=$ $\arg \max \left\{\widehat{\eta}_{v, w, l} \mid v \in[1, V], w \in[1, W], l \in[1, L]\right\}$, where

$v^{*}, w, w^{*}, l^{*}$ and $g^{*}$ mean the resource pattern index, part of partition $\mathbf{q}_{i}$, the index of cell and the group index, respectively. Then the assignment index $\delta_{v^{*}, g^{*}, l^{*}}^{j, i}=1$, the pre-allocated users are $\Upsilon_{\Omega_{g^{*}}}$.

Step3:

Delete $v^{*}$-th row and $w^{*}$-th column from the matrix (31) when $l$ values from 1 to $L$ and put each of it into matrix (32) respectively, then remove users $\Upsilon_{\Omega_{g^{*}}}$ from $\Upsilon$ and update the group set $\left\{\boldsymbol{\Omega}_{m}^{\mathrm{NU}}\right\}$ according to new user set $\boldsymbol{\Upsilon}$, and then, go to step 1 until the elements of matrix(32) are empty. 
TABLE I: COMPLEXITY COMPARISON

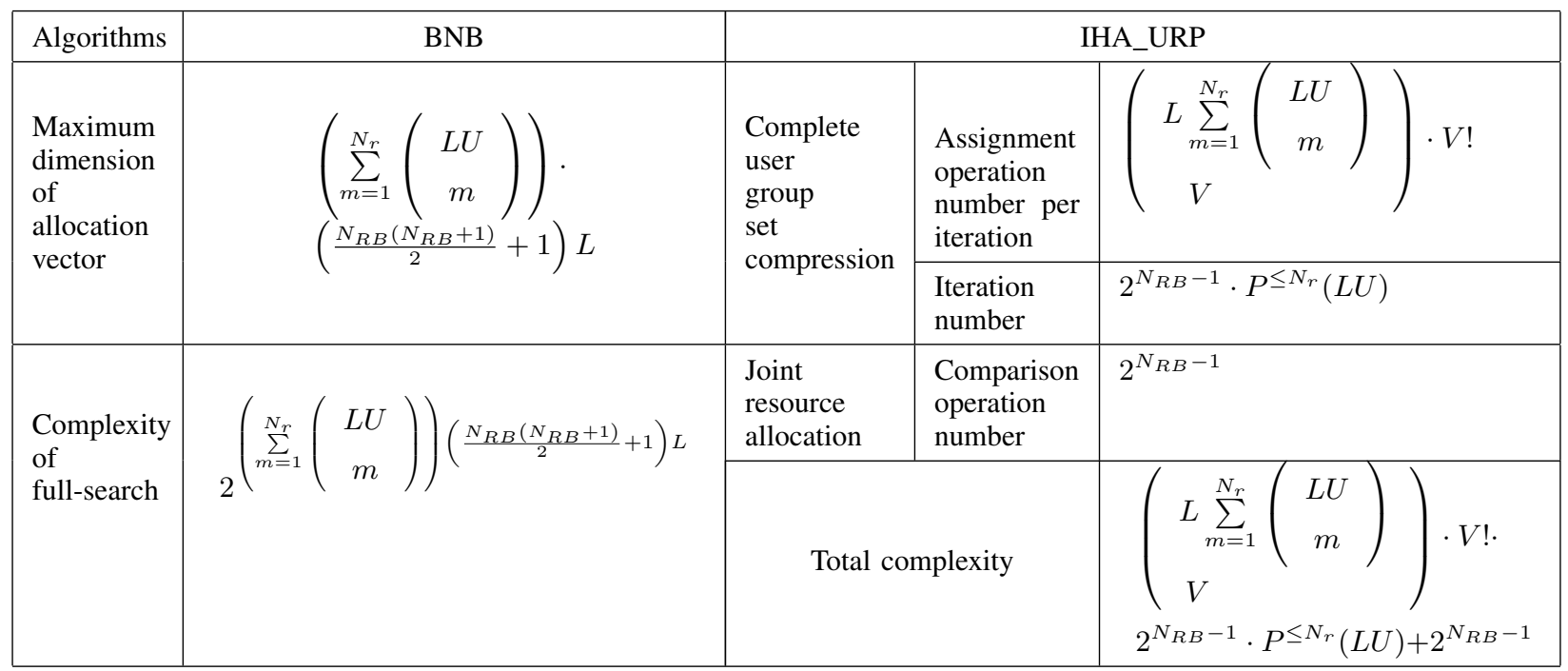

Step4:

Then, the sum-rate of $\mathbf{T}_{j}^{\mathrm{CRP}}$ and $\boldsymbol{\Omega}_{\mathbf{q}_{i}}^{\mathrm{NU}}$ in cell $l$ equals $R_{j, i}=\sum_{v} \sum_{g} \sum_{l} \widehat{\eta}_{v, w, l} \cdot \delta_{v, g, l}^{j, i}$ and the assignment index corresponding to them is $\delta^{j, i}$.

- Iterative Process to Obtain the Resource Allocation:

After $2^{N_{R B}-1} \cdot P \leq N_{r}(L U)$ iterations, we get all best matches between $\left\{\mathbf{T}_{j}^{\mathrm{CRP}}\right\}$ and $\left\{\boldsymbol{\Omega}_{\mathbf{q}_{i}}^{\mathrm{CUG}}\right\}$ for each cell, the sort operation is used to find the resource allocation result:

$$
\left(j^{*}, i^{*}\right)=\underset{j, i}{\arg \max }\left\{R_{j, i} \mid j \in\left[1,\left|\mathbf{T}^{\mathrm{CRP}}\right|\right], i \in[1, n]\right\} .
$$

Then, the maximal sum-rate is $R^{*}=R_{j^{*}, i^{*}}$ and the corresponding resource assignment index $\delta^{*}=\delta^{j^{*}, i^{*}}$ is the solution of joint resource allocation problem (19).

4) Complexity Analysis: The computational complexity of the proposed IHA_URP algorithm comes from two aspects, one is to carry out the complete integer partition operation of $L U$ and $N_{R B}$, which are done only once. The other is to get the optimum resource allocation result based on the partition results of $L U$ and complete RB pattern set.

- Complexity Analysis of integer partitions of $L U$ and $N_{R B}$ for IHA_URP in initial phase:

The Procedure \#1 in APPENDIX A is designed to perform the integer partition operation of $L U$ and obtain the integer partition matrix $\mathbf{Q}$, where the recursive operations are used. So the upper bound of the complexity is given as

$$
T(L U)=1+\sum_{j=1}^{L U-1} T(j)
$$

where $T(L U)$ denotes the number of recursive calls when there are $L U$ users. By assuming that $T(L U)=1$ when $L U=$ 1 and solving the above equation, we can get

$$
T(L U)=2^{L U} .
$$

Similarly, the complexity of integer partition of $N_{R B}$ can be expressed as $T\left(N_{R B}\right)=2^{N_{R B}}$.
- Complexity Analysis of joint resource allocation for IHA_URP in running phase:

According to the analysis above, the operations in initial phase are performed only once, so the running time of the proposed algorithm mainly comes from running phase. In order to evaluate the proposed algorithm, we take the BNB algorithm as a comparison.

Since the accurate complexity analysis of the algorithms solving the joint resource allocation problem is difficult, we use the maximum dimension of allocation vector and the full search number in the optimization to simply analyze the algorithm complexity for BNB algorithm. For proposed IHA_URP algorithm, iterative processes are performed with compression operations where the assignment operations are performed by Hungarian algorithm with greedy strategy. As a combination optimization problem, the complexities of the processes are demonstrated by combination numbers and the iteration number which is given by Lemma 2 and Lemma 3. A comparison of computational complexity between BNB and IHA_URP algorithm is represented in Table I, where $V=\min \left\{N_{R B}, L \sum_{m=1}^{N_{r}}\left(\begin{array}{c}L U \\ m\end{array}\right)\right\}$.

Consider the case that $L=2, N_{R B}=6, U=6$ and $N_{r}=2$. For BNB, the maximum dimension of allocation vector is 3432 and the complexity of full-search is $1.364 \times 10^{1033}$. For the proposed IHA_URP algorithm, the total complexity is $\left(\begin{array}{c}156 \\ 6\end{array}\right) \cdot 6 ! \cdot 32 \cdot 7+32 \approx 2.929 \times 10^{15}$.

From the analysis above, we can find that the proposed IHA_URP algorithm dramatically reduce the complexity. Moreover, since the sub-problems are independent, the solution operations for these $P \leq N_{r}(L U) \times 2^{N_{R B}-1}$ sub-problems can be run in parallel mode for fast processing in practice.

\section{SIMULATION EXPERIMENTS}

\section{A. Simulation Settings}

To evaluate the performance of the proposed dynamic grouping and joint resource allocation algorithm, we conduct 
TABLE II: SIMULATION PARAMETERS

\begin{tabular}{|l|l|l|}
\hline \multirow{4}{*}{$\begin{array}{l}\text { Channel } \\
\text { parameters }\end{array}$} & Channel model: ITU Ped-A & Carrier frequency: $2 \mathrm{GHz}$ \\
\cline { 2 - 3 } & Sampling frequency: $1.92 \mathrm{MHz}$ & Maximum Doppler shift: $10 \mathrm{~Hz}$ \\
\cline { 2 - 3 } & Cell radius:200m & Decay exponent $\gamma: 2$ \\
\cline { 2 - 3 } & \multicolumn{2}{|c|}{ Shadow-fading standard deviation $\sigma_{\text {shadow }: 8 \mathrm{~dB}}$} \\
\hline \multirow{5}{*}{$\begin{array}{l}\text { Simulation } \\
\text { parameters }\end{array}$} & FFT size: 128 & Modulation: MQAM \\
\cline { 2 - 3 } & $N_{R B}: 8$ & $N_{\text {sc }}^{R B}: 12$ \\
\cline { 2 - 3 } & OFDM symbols per frame $: 14$ & RB configure: $12 \times 7$ \\
\cline { 2 - 3 } & Number of cells per cluster $L: 4,3,2,1$ & MIMO detector: MMSE \\
\cline { 2 - 3 } & Average number of users per cell $U: 8$ & BS receiving antenna number $N_{r}: 4$ \\
\cline { 2 - 3 } & UE transmitting antenna number: 1 & Transmission time interval (TTI) duration: $1 \mathrm{~ms}$ \\
\cline { 2 - 3 } & Simulation frames: 1000 & BER Constraint $:$ BER \\
\hline
\end{tabular}

the simulations based on LTE uplink wherein the BS is equipped with four receiving antennas and each user has only one antenna. The simulation parameters are listed in Table II. In addition, we adopt the pedestrian test environment channel A as suggested by ITU-R M.1225 [37]. In the simulations, we use the scenario with perfect power control in one cell, that is, the transmitting power changed adaptively in order to guarantee that the users within a cell will compensate the overall slow fading including path loss and shadowing then obtain the same average channel quality. Then the normalized coefficient of large scale fading is modeled as

$$
\beta_{m, l, u, j}=\frac{z}{\left(d_{l, u, j} / \bar{d}_{l}\right)^{\gamma}},
$$

where $d_{l, u, j}$ is the distance between the $u$-th user of the $j$-th cell to the BS in the $l$-th cell, $\bar{d}_{l}$ is the reference average distance so that the mean of $\beta_{m, l, u, l}$ is $1, \gamma$ is the decay exponent and $z$ is a log-normal random variable, i.e., the quantity $10 \log _{10} z$ is distributed zero-mean Gaussian with standard deviation of $\sigma_{\text {shadow. }}$. In addition, we define the transmitting SNR as $E_{s} / \sigma^{2}$ in these simulations.

The common simulation parameters are illustrated in Table II.

\section{B. Simulation Results}

For comparison purpose, six different joint resource allocation algorithms with user grouping are implemented in the following simulations. The first one is the algorithm with dynamic user grouping proposed in this paper where users in all coordinated cells can be grouped, denoted as 'Dynamic user grouping with multi-cell cooperation'. The second one is the algorithm with dynamic user grouping but without cell cooperation where only users in the same cell can be grouped, denoted as 'Dynamic user grouping without multicell cooperation'. The third one is the algorithm with fixed 2-user grouping which has 32 users in a conventional large cell, i.e. the same number of users compared to multi-cell system with $L=4$, where the large scale fading $\beta=1$ for all users, denoted as 'Conventional fixed 2-user grouping [9]'. The fourth one is the problem with fixed 2-user grouping in conventional large cell solved by Hungarian algorithm in [18], denoted as algorithm 'Conventional fixed 2-user grouping [18]'. The fifth one is the algorithm with fixed 1-user grouping and multi-cell cooperation, denoted as 'No user grouping with multi-cell cooperation'. The last one is the algorithm with fixed 1-user grouping but without multi-cell cooperation, denoted as 'No user grouping without multi-cell cooperation', which is the baseline algorithm serving to show how much improvement can be achieved by our proposed algorithm. In addition, to evaluate the above algorithms in actual systems, grouping criterion (13) is used in above algorithms.

1) The Complexity and Performance of the Proposed IHA_URP Algorithm: For comparison purpose, IHA_URP and BNB algorithms are used to solve the optimization problem (19). In the simulation, MATLAB R2013a is used to run the programs of the proposed IHA_URP algorithm and traditional BNB algorithm for 150 simulation frames with transmitting $\mathrm{SNR}=15 \mathrm{~dB}$.

The comparison for running time between IHA_URP and BNB algorithms is shown in TABLE III. From TABLE III, we can see that with the increase of cell and user numbers, the difference of time complexity between IHA_URP and BNB is getting more and more obvious. When the cluster consists of 1 cell, the run time of those algorithms are close. However, when the cluster consists of 4 cells with 4 users per cell, the running time of BNB is more than 77 times of IHA_URP. In addition, IHA_URP is suitable for fast processing. As mentioned in Section III. C. 4), IHA_URP can be run in parallel mode. Take the case of 4 cells with 6 users per cell in the simulation, the running time of IHA_URP in parallel mode can be reduced to $2486 /(150 \times 351 \times 128) \approx 0.00037 \mathrm{~s}$ per frame, which is less than one TTI.

To evaluate the performance loss of IHA_URP, we conduct the simulation for 1000 simulation frames. The simulation parameters and results are shown in TABLE IV, where $p l r=$ $\frac{S E_{B N B}-S E_{I H A} U R P}{S E_{B N B}}$ is the performance loss rate of IHA_URP, $S E_{B N B}$ and $S E_{I H A_{-} U R P}$ denote the spectrum efficiency obtained by BNB and IHA_URP respectively. From TABLE IV, we can see that the performance of IHA_URP is close to that of $\mathrm{BNB}$ which has the optimal performance. From TABLE III and IV, we observe that IHA_URP can achieve 
TABLE III: RUNNING TIME (second) COMPARISON OF BNB AND IHA_URP(SNR=15dB)

\begin{tabular}{|c|c|c|c|c|c|c|c|c|c|c|c|c|}
\hline \multirow{2}{*}{$\begin{array}{l}\text { Users } \\
\text { per } \\
\text { cell }\end{array}$} & \multicolumn{3}{|c|}{1 cell } & \multicolumn{3}{|c|}{2 cells } & \multicolumn{3}{|c|}{3 cells } & \multicolumn{3}{|c|}{4 cells } \\
\hline & $\begin{array}{l}\text { IHA_ }_{-} \\
\text {URP }\end{array}$ & BNB & ratio & $\begin{array}{l}\text { IHA_ } \\
\text { URP }\end{array}$ & BNB & ratio & $\begin{array}{l}\text { IHA_ } \\
\text { URP }\end{array}$ & BNB & ratio & $\begin{array}{l}\text { IHA_ } \\
\text { URP }\end{array}$ & BNB & ratio \\
\hline 2 users & 9.08 & 10.88 & 1.20 & 20.72 & 28.66 & 1.38 & 47.62 & 84.88 & 1.78 & 271.64 & 437.94 & 1.61 \\
\hline 4 users & 11.15 & 15.17 & 1.36 & 57.52 & 109.79 & 1.91 & 198.46 & 1370.99 & 6.91 & 683.8622 & 52719.49 & 77.09 \\
\hline 6 users & 14.57 & 27.03 & 1.86 & 109.91 & 559.13 & 5.09 & 598.14 & 25088.32 & 41.94 & 2480.39 & $*$ & $*$ \\
\hline
\end{tabular}

Note: '*' means the results are difficult to be obtained due to high complexity.

TABLE IV: SPECTRUM EFFICIENCY PERFORMANCESOF BNB AND IHA_URP (bit/s/Hz)

\begin{tabular}{|c|c|c|c|c|c|c|}
\hline Cells per cluster & Users per cell & Algorithms & $\mathrm{snr}=3$ & $\mathrm{snr}=9$ & $\mathrm{snr}=15$ & $\mathrm{snr}=21$ \\
\hline \multirow{6}{*}{1 cell } & \multirow{3}{*}{2 users } & $\mathrm{BNB}$ & 1.38559238 & 3.06559525 & 5.20677581 & 7.91926021 \\
\hline & & IHA_URP & 1.38557823 & 3.06558404 & 5.20677581 & 7.91926021 \\
\hline & & $p l r\left(10^{-5}\right)$ & 1.02 & 3.66 & 0 & 0 \\
\hline & \multirow{3}{*}{4 users } & $\mathrm{BNB}$ & 1.84337122 & 3.81544563 & 6.24504865 & 9.89343769 \\
\hline & & IHA_URP & 1.84327065 & 3.81539657 & 6.24503353 & 9.89343769 \\
\hline & & $p l r\left(10^{-5}\right)$ & 5.46 & 1.29 & 0.24 & 0 \\
\hline \multirow{6}{*}{2 cells } & \multirow{3}{*}{2 users } & $\mathrm{BNB}$ & 2.017984 & 4.07035328 & 7.42745305 & 11.05374851 \\
\hline & & IHA_URP & 2.01793915 & 4.07033781 & 7.42745305 & 11.05374851 \\
\hline & & $p \operatorname{lr}\left(10^{-5}\right)$ & 2.22 & 3.80 & 0 & 0 \\
\hline & \multirow{3}{*}{4 users } & $\mathrm{BNB}$ & 2.28317308 & 4.91721589 & 8.77243234 & 13.07939894 \\
\hline & & IHA_URP & 2.28313125 & 4.91704633 & 8.77240121 & 13.07936074 \\
\hline & & $p \operatorname{lr}\left(10^{-5}\right)$ & 1.83 & 3.45 & 0.36 & 0.29 \\
\hline \multirow{6}{*}{3 cells } & \multirow{3}{*}{2 users } & BNB & 2.28614565 & 4.27463884 & 7.01307279 & 10.715384 \\
\hline & & IHA_URP & 2.28605684 & 4.27455149 & 7.01306356 & 10.7153708 \\
\hline & & $p \operatorname{lr}\left(10^{-5}\right)$ & 3.88 & 2.04 & 0.13 & 0.12 \\
\hline & \multirow{3}{*}{4 users } & $\mathrm{BNB}$ & 3.41562294 & 5.15118125 & 9.19341369 & 13.93403285 \\
\hline & & IHA_URP & 3.41550878 & 5.15104525 & 9.19339925 & 13.93391580 \\
\hline & & $\operatorname{plr}\left(10^{-5}\right)$ & 3.34 & 2.64 & 0.16 & 0.84 \\
\hline \multirow{6}{*}{4 cells } & \multirow{3}{*}{2 users } & $\mathrm{BNB}$ & 3.07245208 & 6.09230888 & 9.9807998 & 14.94322085 \\
\hline & & IHA_URP & 3.07237348 & 6.09196464 & 9.98071122 & 14.94319019 \\
\hline & & $p \operatorname{lr}\left(10^{-5}\right)$ & 2.56 & 5.65 & 0.89 & 0.21 \\
\hline & \multirow{3}{*}{4 users } & $\mathrm{BNB}$ & $*$ & $*$ & $*$ & $*$ \\
\hline & & IHA_URP & 4.38683954 & 8.04567089 & 13.06021382 & 19.12991122 \\
\hline & & $p l r\left(10^{-5}\right)$ & $*$ & $*$ & $*$ & $*$ \\
\hline
\end{tabular}

Note: '*' means the results are difficult to be obtained due to high complexity.

the close-to-optimal performance with much lower complexity than BNB. Since BNB algorithm is difficult to implement, IHA_URP algorithm is used to demonstrate the performance of the joint resource allocation in the following simulations.

2) The Impact of User Grouping for Multi-cell Systems: To compare the proposed algorithm with fixed user grouping algorithms and other conventional joint user pairing and resource allocation algorithms, we simulate fixed 1-, 2-, 3-user grouping algorithms and the modified algorithms proposed in [9] and [18] by using AM techniques respectively, that is, replace the Shannon capacity with AM rate of Eq. (11). Figure 4(a) and (b) show the spectrum efficiency versus transmitting SNR for different grouping algorithms. In addition, in order to reflect the trade-off between BER performance and spectral efficiency for the proposed algorithm, we set different BER constraints with $L=4$. The results are shown in Figure 4(c). 


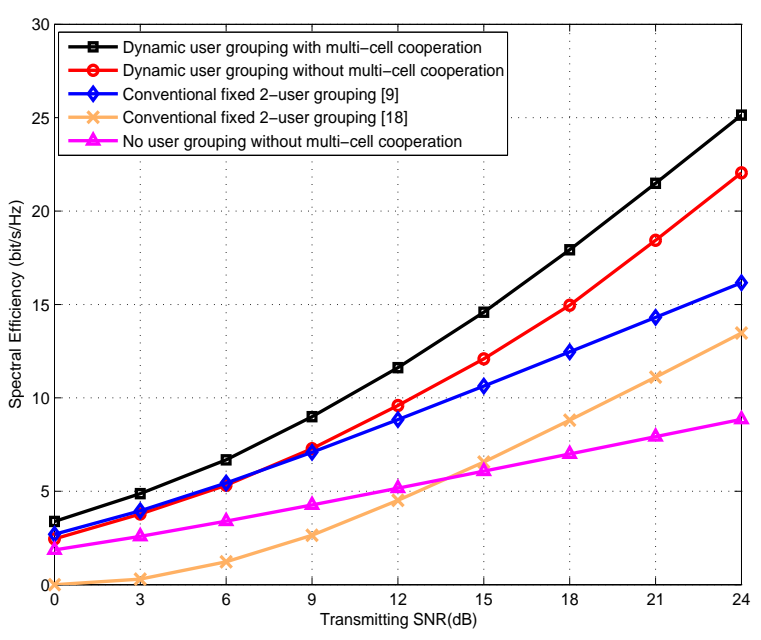

(a) Different resource allocation algorithms

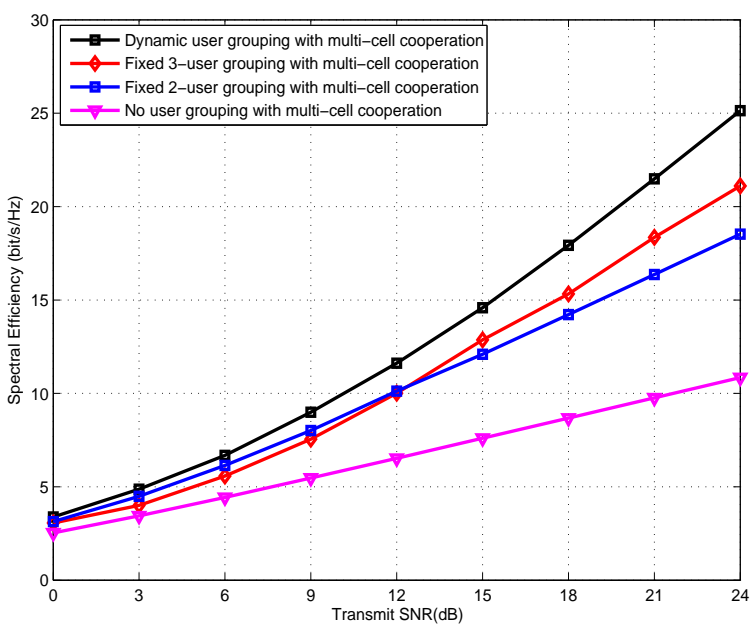

(b) Fixed user grouping algorithms

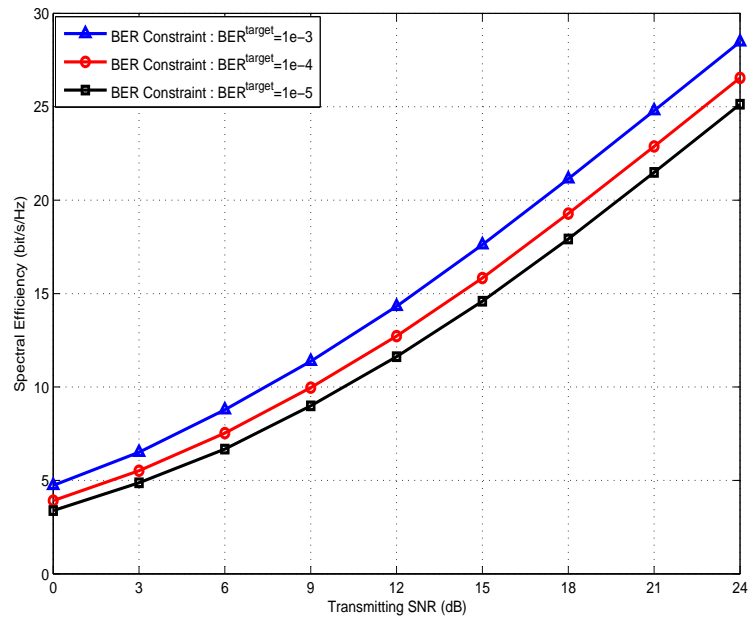

(c) Different BER constraints

Fig. 4: Spectral efficiency versus transmitting SNR for different resource allocation algorithms and different BER constraints
From Figure 4(a), we can see that 'Dynamic user grouping with multi-cell cooperation' algorithm outperforms 'Dynamic user grouping without multi-cell cooperation' algorithm and 'No user grouping without multi-cell cooperation' algorithm, which due to the former can achieve multi-cell selective gain and grouping multiplexing gain while the latter two algorithms cannot. The 'Dynamic user grouping with multicell cooperation' algorithm achieves much higher spectral efficiency than the 'Conventional fixed 2-user grouping [9]' algorithm and 'Conventional fixed 2-user grouping [18]' algorithm. The main reason is that the latter two algorithms can only have fixed 2-user grouping regardless of the value of SNR while the former has 2-user grouping in low SNR region and has $N_{r}$-user grouping in high SNR region which achieves grouping multiplexing gain and multi-cell selective gain. In addition, any number of consecutive RBs are assigned to one user group in 'Dynamic user grouping with multicell cooperation' algorithm and 'Conventional fixed 2-user grouping [9]' algorithm while one RB is assigned to one user group in 'Conventional fixed 2-user grouping [18]' algorithm, so the former two algorithms obtain better RB selective gain than the latter.

From Figure 4(b), we can see that the spectral efficiency of 'Dynamic user grouping with multi-cell cooperation' algorithm always outperforms that of fixed 1-, 2-, 3-user grouping algorithms in both high SNR region and low SNR region. The performance gain comes from grouping multiplexing gain and multiuser selection gain. In the low SNR region, due to the BER constraint, the algorithms of small fixed grouping user number work well while the algorithms of large fixed grouping user number work well in high SNR region.

From Figure 4(c), we can see that the spectral efficiency rises when BER performance constraint drops, which mainly because the number of active grouping users becomes large. This leads to different spectral efficiency due to the different grouping user multiplexing gain and multiuser selection gain. So, the proposed 'Dynamic user grouping with multi-cell cooperation' algorithm can achieve trade-off between BER performance and spectral efficiency as required.

3) The Impact of Cell Number in One Cluster: In this simulation, we set $L=1,2,3$ and 4 respectively and use the 'Dynamic user grouping with multi-cell cooperation' algorith$\mathrm{m}$ and 'Fixed 2-user grouping with multi-cell cooperation' algorithm to evaluate the impact of cell number in one cluster. To ensure the fairness, we let each cell contains $\hat{N}=\frac{N_{R B}}{L}$ RBs so that one cluster has the same number of RBs in different experiments. In Figure 5, we show the spectrum efficiency versus transmitting SNR for one cluster consisting of different number of cells.

From Figure 5, we can see both the algorithms with dynamic user grouping and the algorithms with fixed 2-user grouping have the phenomenon that spectral efficiency of the cluster consisting of 4 cells outperforms that of others. The conclusion is obtained that the more cells one cluster consists, the higher spectral efficiency is. Because the cluster consisting of more cells can achieve much higher RB selection gain, multiuser selection gain and multi-cell selection gain than the cluster with less cells. For example, the cluster consisting of 4 cells 
has 32 users for grouping and $8 \mathrm{RBs}$ for allocation which can achieve much higher selection gains than the cluster consisting of only 1 cell case (i.e. the latter only has 8 users and 2 RBs).

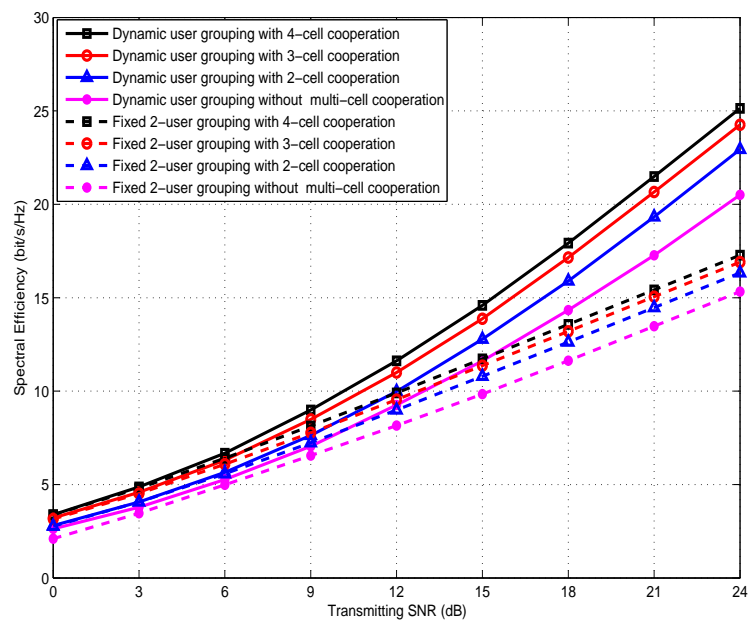

Fig. 5: Spectral efficiency versus transmitting SNR for one cluster consisting of different number of cells

4) The Impact of User Number: In this simulation, we set $L=4$ and perform the 'Dynamic user grouping with multicell cooperation' algorithm with different user numbers. The results are shown in Figure 6.

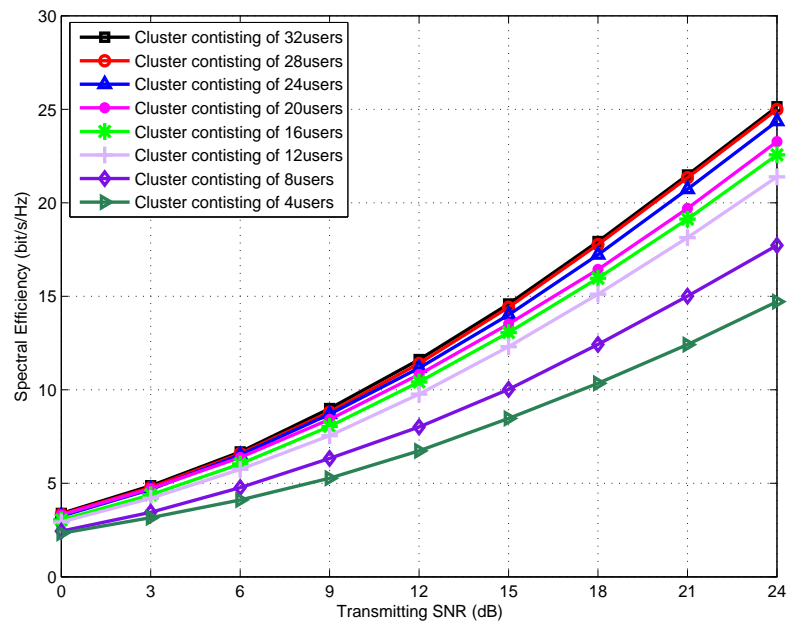

Fig. 6: Spectral efficiency versus transmitting SNR for one cluster consisting of different user numbers

From Figure 6, we can see that the spectrum efficiency increases as the number of users increases. As expected, when the number of users increases, the overall spectral efficiency of each experiment improves due to the increased degrees of user freedom. Meanwhile, it can be seen from the figure that as the number of users in one cluster increases over 28 , the increase of spectrum efficiency gain becomes negligible.
5) Fairness Evaluation of the Proposed Algorithm with Proportional Scheduling: The different algorithms using weighted fairness factor of Eq. (14) are compared in this simulation. To evaluate fairness performance, we adopt the Jain's fairness index [38] as follows:

$$
J F=\left(\sum_{i=1}^{N} x_{i}\right)^{2} / N \sum_{i=1}^{N} x_{i}{ }^{2} .
$$

We set transmitting $\mathrm{SNR}=15 \mathrm{~dB}$ and simulate the fairness index and spectral efficiency when the smoothing window length $T_{W}$ ranges from 10 to 110 . The results are shown in Figure 7(a) and 7(b).

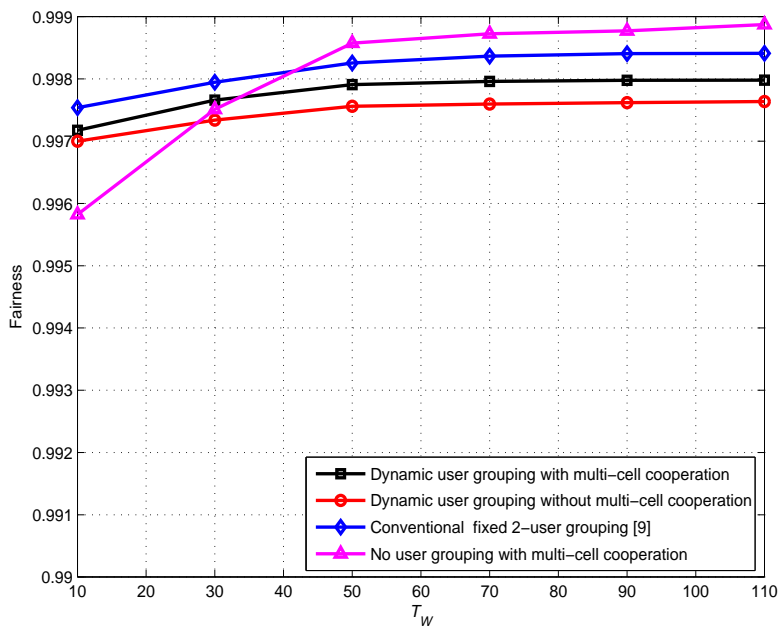

(a) Fairness index

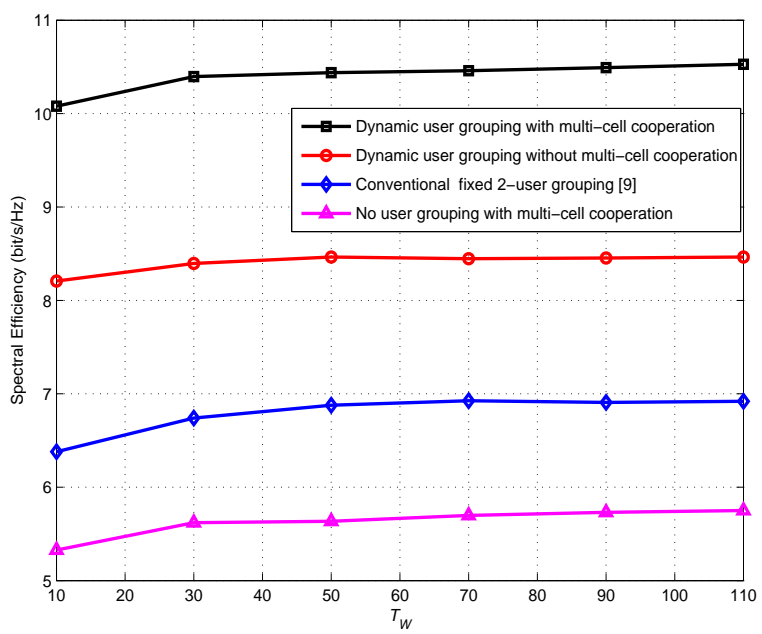

(b) Spectral efficiency

Fig. 7: Fairness index and spectral efficiency versus smoothing window length $T_{\mathrm{W}}$ for different resource allocation algorithms using weighted fairness factor

From Figure 7(a), we can see that the fairness indices of those four algorithms are greater than 0.99 and close to 1 . So when user fairness is taken into account by using the proportional scheduling, the fairness of each user in the system is guaranteed. On the other hand, from Figure 7(b), we can see 
that the 'Dynamic user grouping with multi-cell cooperation' algorithm has much larger spectral efficiency than 'Dynamic user grouping without multi-cell cooperation', 'Conventional fixed 2-user grouping [9]' and 'No user grouping with multicell cooperation' algorithms no matter smoothing window length is small or large. So the proposed algorithm has better trade-off between user fairness and spectral efficiency than others.

In addition, from Figure 7(a) and 7(b), we can see that both fairness index and spectral efficiency curves for all algorithms tend to be stabilized when the length of smoothing window is greater than 70 . So, it is suitable to set the smoothing window length 70 for practical use.

\section{CONCLUSIONS}

This paper proposed a dynamic user grouping and joint resource allocation algorithm for SC-FDMA uplink in multicell virtual MIMO systems. We derived the dynamic multicell user grouping criteria and proposed a novel joint resource allocation algorithm with multi-cell cooperation to achieve maximum system overall throughput with MMSE equalization and AM techniques. In addition, to reduce the computation complexity, we proposed an efficient IHA_URP algorithm for solution of the joint resource allocation problem. The simulation results demonstrated that the proposed algorithm attains better system throughput than both the traditional algorithms with fixed user grouping and the algorithms without multi-cell cooperation.

\section{APPENDIX A}

PROCEDURES FOR GENERATION OF COMPLETE USER GROUP SET $\boldsymbol{\Omega}^{\mathrm{CUG}}$

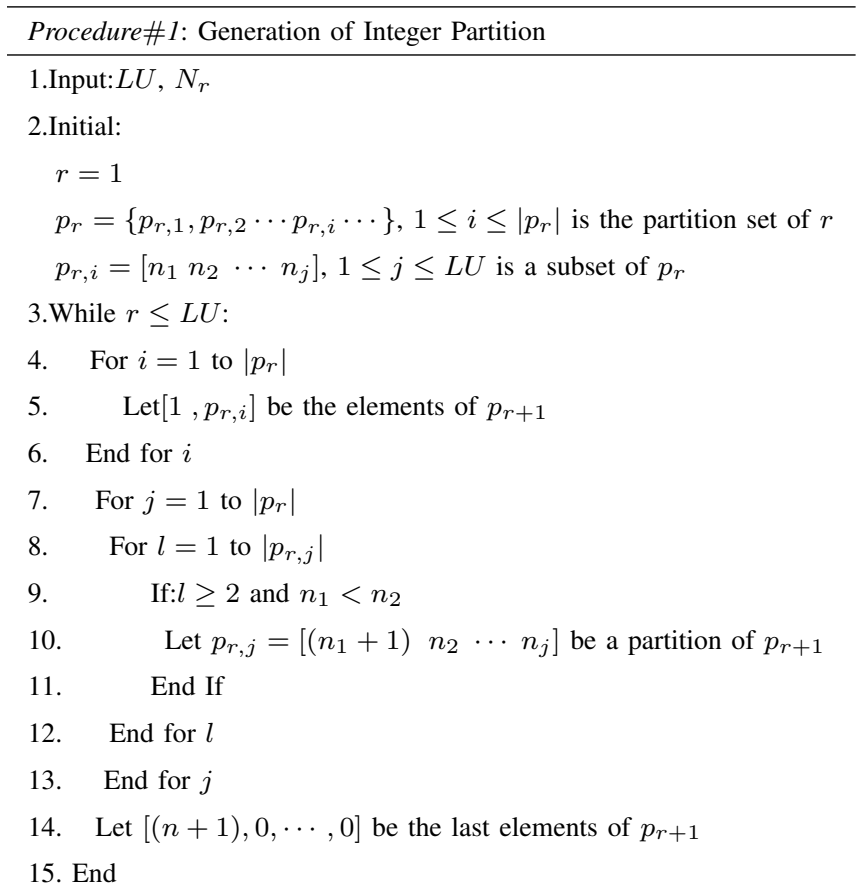

16. Delete the subsets of $p_{L U+1}$ contained elements greater than $N_{r}$ and put the rest of subsets into matrix $\mathbf{Q}$

17. Output Q

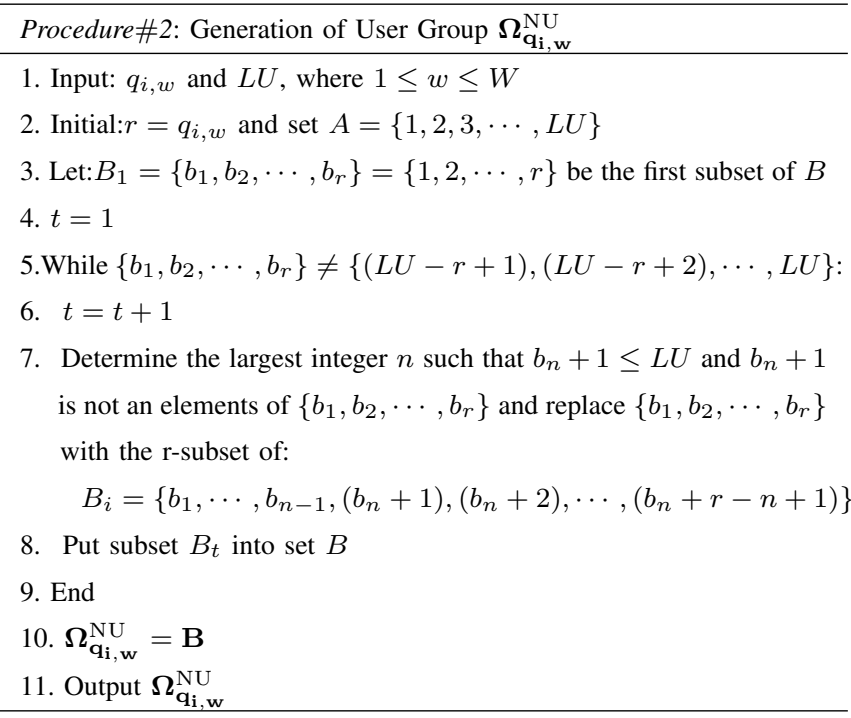

\section{REFERENCES}

[1] A. J. Paulraj, D. A. Gore, R. U. Nabar, and H. Bolcskei, "An overview of MIMO communications - a key to gigabit wireless," Proc. IEEE, vol. 92, no. 2, pp. 198-218, Feb. 2004.

[2] 3GPP TSG-RAN1, R1-0501162, "UL virtual MIMO transmission for E-UTRA," San Diego, USA, Oct. 2005.

[3] J. Duplicy, B. Badic, R. Balraj, R. Ghaffar, and P. Horvath, "MUMIMO in LTE systems," EURASIP J. Wireless Commun. Netw., vol. 2011: 496763.

[4] S. Sesia, I. Toufik, and M. Baker, The UMTS Long Term Evolution: from theory to practice. New York: John Wiley \& Sons, 2009.

[5] 3GPP TSG RAN1 \#46, R1-062074, "Link simulation results for uplink virtual MIMO," Tallinn, Estonia, Aug. 2006.

[6] H. Zhao, S. Ma, F. Liu, and Y. Tang, "A Suboptimal Multiuser Pairing Algorithm with Low Complexity for Virtual MIMO Systems," IEEE Trans. Veh. Tech., vol. 63, no. 7, pp. 3481-3486, Jan. 2014.

[7] A. Goldsmith, S. A. Jafar, N. Jindal, and S. Vishwanath, "Capacity limits of MIMO channels," IEEE J. Select. Areas Commun., vol. 21, no. 5, pp. 684-702, June 2003.

[8] 3GPP TSG-RAN1 \#46, R1-062052, "UL system analysis with SDMA," Tallinn, Estonia, Aug. 2006.

[9] J. Fan, G. Y. Li, Q. Yin, B. Peng, and X. Zhu, "Joint User Pairing and Resource Allocation for LTE Uplink Transmission," IEEE Trans. Wireless Commun., vol. 11, no. 8, pp. 2838-2847, Aug. 2012

[10] B. Fan, W. Wang, Y. Lin, L. Huang, and K. Zheng, "Spatial Multi-User Pairing for Uplink Virtual-MIMO Systems with Linear Receiver," in Proc. IEEE WCNC, Budapest, pp. 1807-1811, Apr. 2009.

[11] X. Chen, H. Hu, H. Wang, H. Chen, and M. Guizani, "Double proportional fair user pairing algorithm for uplink virtual MIMO systems," IEEE Trans. Wireless Commun., vol. 7, no. 7, pp. 2425C2429, July 2008.

[12] 3Gpp TSG-RAN1 WG1 \#43, "UL Virtual MIMO System Level Performance Evaluation for E-UTRA," R1-051422, Seoul, Korea, 7th-11th Nov. 2005.

[13] S. Dhakal, and K. Joonbeom, "Statistical analysis of user-pairing algorithms in virtual MIMO systems," Wireless Telecommunications Symposium (WTS), Tampa, pp. 1-5, April 2010.

[14] Y. Li, W. Wang, X. Zhang, and M. Peng, "Combined Proportional Fair and Maximum Rate Scheduling for Virtual MIMO," in Proc. IEEE Veh. Tech.Conf. , Calgary, pp. 1-4, Sept. 2008.

[15] X. Wang, W. Wang, Z. Zhao, and T. Zhu, "Fairness Adjustable Grouping Multiuser Scheduling for MIMO MAC with MMSE-SIC Receiver," IEEE GLOBECOM Workshops, New Orleans, pp. 1-5, Nov. 30, Dec. 2008.

[16] 3GPP TSG-RAN1 \#46, R1-062052, "UL system analysis with SDMA," Tallinn, Estonia, Aug. 2006. 
[17] O. B. Karimi, M. A. Toutounchian, J. Liu, and C. Wang, "Lightweight User Grouping with Flexible Degrees of Freedom in Virtual MIMO," IEEE J. Select. Areas Commun., vol. 31, no. 10, pp. 2004-2012, Oct. 2013.

[18] M. A. Ruder, D. Ding, U. L. Dang, A. V. Vasilakos, and W. H. Gerstacker, "Joint user grouping and frequency allocation for multiuser SC-FDMA transmission," Physical Communication, vol. 8, pp. 91-103, 2013.

[19] 3GPP, "TSG-RAN EUTRA, rel.10", TR 36.213, Dec. 2010.

[20] I. C. Wong, O. Oteri, and W. McCoy, "Optimal resource allocation in uplink SC-FDMA systems," IEEE Trans. Wireless Commun., vol. 8, no. 5, pp. 2161C2165, May 2009.

[21] N. Prasad, H. Zhang, H. Zhu, and S. Rangarajan, "Multi-User MIMO Scheduling in the Fourth Generation Cellular Uplink," IEEE Trans. Wireless Commun., vol. 12, no. 9, pp. 4272-4285, Sept. 2013.

[22] H. Kuhn, "The Hungarian method for the assignment algorithm, "Naval Research Logistics Quarterly, vol. 1, no. 1-2, pp. 83-97, 1955.

[23] H. Kuhn, "Variants of the Hungarian method for the assignment problem, " Naval Research Logistics Quarterly, vol. 3, pp. 253-258, 1956.

[24] X. F. Lu, K. Yang, W. N. Li, S. J. Qiu, and H. L. Zhang, "Joint user grouping and resource allocation for uplink virtual MIMO systems," Science China Information Sciences, vol. 59, pp. 1-14, 2016.

[25] G. Y. Li, J. Niu, D. Lee, J. Fan, and Y. Fu, "Multi-Cell Coordinated Scheduling and MIMO in LTE," IEEE Commun. Surveys Tutorials, vol. 16, no. 2, second quarter 2014.

[26] G. Nauryzbayev, E. Alsusa, "Enhanced Multiplexing Gain Using Interference Alignment Cancellation in Multi-Cell MIMO Networks," IEEE Trans. Inform. Theory, vol. 62, no. 1, Jan. 2016.

[27] K. Pham, K. Lee, "Interference Alignment for Multicell Multiuser MIMO Uplink Channels," IEEE Trans. Veh. Tech., vol. 65, no. 7, July 2016.

[28] I. Choi and C. Lee, "A Scaled Hard Interference Cancellation Scheme in an Uplink Multicell Environment," IEEE Trans. Veh. Tech., vol. 64, no. 12, Dec. 2015.

[29] Q. Yang, T. Jiang, C. Jiang, Z. Han, and Z. Zhou ,"Joint Optimization of User Grouping and Transmitter Connection on Multi-Cell SNR Blind Interference Alignment," IEEE Access, vol. 4, no. 1, pp. 4974-4988, Apr. 2016.

[30] P. Li and R. C. Lamare, "Distributed Iterative Detection With Reduced Message Passing for Networked MIMO Cellular Systems," IEEE Trans. Veh. Tech., vol. 63, no. 6, July 2014.

[31] J. Zhang, R. Chen, J. G. Andrews, A. Ghosh and R. W. Heath, "Networked MIMO with Clustered Linear Precoding," IEEE Trans. Wireless Commun., vol. 8, no. 4, Apr. 2009.

[32] H. Q. Ngo, E. G. Larsson, and T. L. Marzetta, "The Multi-cell Multiuser MIMO Uplink with Very Large Antenna Arrays and a FiniteDimensional Channel," IEEE Trans. Commun., vol. 61, pp. 2350-2361, June 2013.

[33] A. Goldsmith, S. Chua, "Variable-rate variable-power MQAM for fading channels," IEEE Trans. Commun., vol. 45, no. 10, pp. 1218C1230, 1997.

[34] M. Kobayashi and G. Caire, "Joint beamforming and scheduling for a multi-antenna downlink with imperfect transmitter channel knowledge," IEEE J. Sel. Areas Commun., vol. 25, no. 7, pp. 1468C1477, Sep. 2007.

[35] http://www.mathworks.co.uk/help/optim/examples/binary-integerprogramming.html, Dec. 2012.

[36] K. G. Murty, Linear programming. New York: John Wiley \& Sons, 1983.

[37] Recommendation ITU-R M.1225, "Guidelines for evaluation of radio transmission technologies for IMT-2000," Int. Telecommun. Union, 1997.

[38] R. Jain, D. Chiu, W. Hawe, "A quantitative measure of fairness and discrimination for resource allocation in shared computer systems," $D E C$ Research Report Tr-301, 1984.

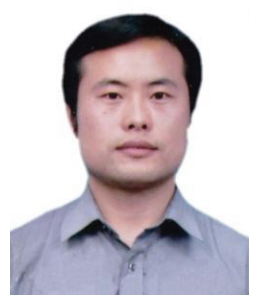

Xiaofeng $\mathbf{L u}$ received the Ph.D. in Communication and Information systems from Huazhong University of Science and Technology, Wuhan, China, in 2006, and the MSc and BSc degree from HuNan University Changsha, China, in 1999 and SiChuan University, Chengdu, China, in 1996, respectively. From 1999 to 2003, he was a R\&D engineer in Wuhan Research Institute of Post and Telecommunications. He is currently an Associate Professor of the State Key Laboratory of Integrated Service Networks at Xidian University, Xian, China. His main research interests lie in the area of broadband wireless communications, covering topics such as resource allocation and virtualization, MU-MIMO, and OFDMA.

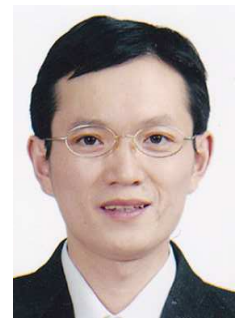

Qiang Ni (M'04-SM'08) received the Ph.D. degree in engineering from Huazhong University of Science and Technology, Wuhan, China. He is currently a Full Professor and the Head of Communication Systems Research Group at the School of Computing and Communications, Lancaster University, Lancaster, UK. His main research interests lie in the area of future generation communications and networking systems, including energy and spectrum efficient green wireless communications, NonOrthogonal Multiple Access (NOMA), 5G, massive MIMO, SDN, game theory, heterogeneous networks, cognitive radio network systems, cloud networks, energy harvesting, IoTs, vehicular networks, big data analytics, etc. He had published more than 180 research papers in international journals and conferences. He was an IEEE 802.11 Wireless Standard Working Group Voting Member and a Contributor to the IEEE Wireless Standards.

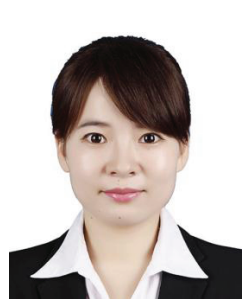

Wenna Li was born in Shaanxi, China. She received the BSc degree in communication engineering from Changan University, Xian, China, in 2014. She is currently pursuing the MSc degree in communication and information system in the State Key Laboratory of Integrated Service Networks at Xidian University, Xian, China. Her research interests include SC-FDMA, virtual MIMO and resource allocation of wireless communications.

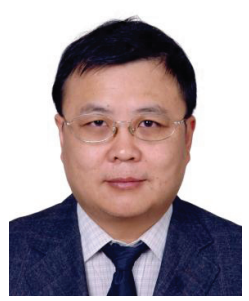

Hailin Zhang received the Ph.D. from Xidian University, Xian, China, in 1991, and his B.S. and M.S. degrees from Northwestern Polytechnic University, Xian, China, in 1985 and 1988 respectively. He is currently a Full Professor and the Head of School of Telecommunications Engineering at Xidian University, Xian, China. His main research interests lie in the area of broadband wireless communications, including massive MIMO, OFDM, space-time coding, etc. $\mathrm{He}$ has recently published 78 papers in telecommunications journals and proceedings. 